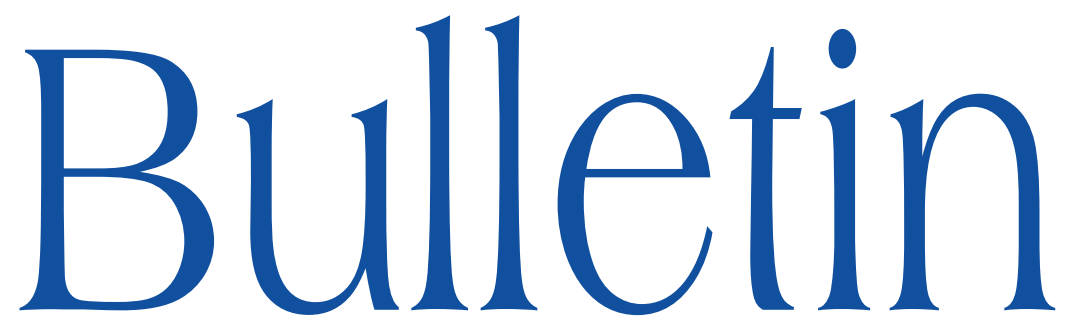

de la SOCIÉTÉ MATHÉMATIQUE DE FRANCE

\title{
COMPORTEMENT HARMONIQUE DES DENSITÉS CONFORMES ET FRONTIÉRE DE MARTIN
}

\author{
Thomas Roblin
}

\section{Tome 139}

Fascicule 1

2011 


\title{
COMPORTEMENT HARMONIQUE DES DENSITÉS CONFORMES ET FRONTIÈRE DE MARTIN
}

\author{
PAR THOMAS ROBLIN
}

\begin{abstract}
RÉsumé. - Traitant la série de Poincaré d'un groupe discret d'isométries en courbure négative comme un noyau de Green, on établit une théorie du potentiel assez comparable à la théorie classique pour affirmer un parallèle entre densités conformes à la Patterson-Sullivan et densités harmoniques, et notamment définir une frontière de Martin où les densités ergodiques forment la partie minimale, et enfin l'identifier géométriquement sous hypothèse d'hyperbolicité.
\end{abstract}

Abstract (Harmonic behaviour of conformal densities and Martin boundary)

By treating the Poincaré series of a discrete group of isometries in negative curvature like a Green kernel, we set up a potential theory enough comparable to the classical theory, which allows us to draw a parallel between conformal densities and harmonic densities, and in particular to define a Martin boundary in which ergodic densities make up the minimal part, and even to give a geometrical identification of it under a hyperbolicity assumption.

Texte reçu le 30 avril 2009, révisé les 20 octobre 2009 et 15 décembre 2009, accepté le 12 octobre 2010

Thomas Roblin, CNRS UMR 7599, Laboratoire de Probabilités et Modèles aléatoires, Université Pierre et Marie Curie-Boîte courrier 188, 75252-Paris Cedex 05, France • E-mail : thomas.roblin@upmc.fr

Classification mathématique par sujets (2000). - 37F35, 20F65, 20F67, 22E40, 31C35, 31E99.

Mots clefs. - Mesures de Patterson-Sullivan, groupes discrets, courbure négative, théorie du potentiel, frontière de Martin, groupes hyperboliques. 


\section{Introduction}

Le présent article prolonge l'idée de départ de [20], en développant dans une autre voie les conséquences de ce qu'on y avait appelé le principe des ombres. Là il s'agissait d'appliquer ce principe à la théorie ergodique des revêtements galoisiens nilpotents des variétés convexe-cocompactes; ici, l'on poursuit davantage le parallèle amorcé entre densités conformes à la Patterson-Sullivan et densités harmoniques.

Les densités conformes ou de Patterson-Sullivan, qui prennent leur origine dans [17] et [21], ont permis d'étendre largement la théorie ergodique des groupes d'isométries discrets sur les espaces à courbure strictement négative, en se substituant à la densité de Lebesgue utilisée dans le cadre classique des groupes de revêtements des surfaces compactes à courbure constante. Cette dernière coïncide par ailleurs avec la densité harmonique liée au laplacien (hyperbolique), et de ce fait appelle aux concepts et aux techniques de la théorie du potentiel, qui sont au cour de [22], [14], [2] entre autres. Mais si dans un cadre un peu plus large, les densités harmoniques attachées au laplacien restent présentes, elles s'avèrent néanmoins généralement distinctes des densités conformes (voir [13]). Aussi la question se pose-t-elle de savoir si les densités conformes peuvent apparaître comme harmoniques pour un autre opérateur (loin d'être régulier en général), disons plutôt pour une autre théorie du potentiel. Par exemple, dans [9], pour certains groupes (groupes de type divergent), et pour la densité (alors unique) de dimension égale à l'exposant critique, ce programme a été réalisé dans une version discrète en produisant une marche aléatoire sur le groupe pour laquelle la mesure de Patterson-Sullivan est harmonique; cette construction permet l'identification du bord de Poisson, mais reste décevante à d'autres égards : perte de symétrie, absence de moment exponentiel comme on en trouve dans la discrétisation du laplacien classique (cf [4]).

Dans cet article, nous apportons une réponse différente : nous sommes capables de rebâtir une bonne part de l'ossature de la théorie du potentiel pour les densités conformes, et ce dans une grande généralité, sans pour autant parvenir à lui donner pour fondement un opérateur ou noyau résolvant précis. Aussi nous tiendrons-nous plutôt dans une analogie entre densités conformes et harmoniques, qui fera notamment apparaître la série de Poincaré du groupe comme fonction de Green. Des propriétés essentielles seront mises en lumière : théorème de Fatou, réduite (balayage), principe du maximum, équation résolvante, mesure harmonique des boules, mais le tout dilaté par des constantes comme si l'on avait effectué une discrétisation grossière. En particulier, nous introduirons une frontière de Martin pour les densités conformes, intéressante d'ailleurs en regard de la construction initiale de Patterson dans [17], et qui 
sera identifiée géométriquement dans certains cas. Quant aux groupes compris dans cette étude, nous n'exigeons d'eux qu'une certaine uniformité que traduit le principe des ombres ci-dessous; sont par exemple concernés tous les sous-groupes distingués des groupes convexe-cocompacts.

$\mathrm{Au}$ terme de ce travail, nous reviendrons sur le problème laissé ouvert, mais considérablement précisé, de trouver une théorie du potentiel vraiment exacte pour les densités conformes.

Signalons, avant de commencer l'exposition, que nous nous sommes ici tenus aux densités conformes sur des espaces CAT $(-1)$ par souci de simplicité, mais que ce cadre axiomatique aurait encore pu être étendu (mesures de Gibbs, mesures quasi-conformes).

Nous tenons enfin à remercier le rapporteur anonyme pour sa lecture attentive du manuscrit.

\section{Préliminaires}

2.1. Géométrie. - Nous considérerons un espace métrique CAT $(-1)$ propre $X$. Nous renvoyons par exemple à [6], [11], [3] et à [19] pour les définitions et les premières propriétés de cette catégorie d'espaces généraux, dont il suffira au lecteur de savoir qu'elle comprend les variétés riemanniennes complètes simplement connexes à courbures sectionnelles $\leq-1$, et également les arbres et les immeubles hyperboliques. Nous noterons $\partial X$ le bord visuel de $X$, et $\bar{X}=X \cup \partial X$. Pour $x, y \in X$, nous désignerons par $d(x, y)$ la distance de $x$ à $y$.

Nous examinerons un groupe discret $\Gamma$ d'isométries de $X$ fixé, dont l'ensemble limite sera noté $\Lambda(\Gamma)$. Nous supposerons que $\Gamma$ n'est pas élémentaire, c'est-à-dire qu'il ne laisse invariant aucun sous-ensemble fini de $\bar{X}(\Lambda(\Gamma)$ est alors le plus petit fermé invariant par $\Gamma$, et est infini). Nous noterons $X_{\Gamma}$ l'enveloppe géodésique de l'ensemble limite, c'est-à-dire la réunion dans $X$ des géodésiques dont les extrémités sont dans $\Lambda(\Gamma)$. Alors $\overline{X_{\Gamma}}$ est un fermé de $\bar{X}$ invariant par $\Gamma$, qui représentera ici une approximation convenable de l'enveloppe convexe de $\Lambda(\Gamma)$ (grâce à des arguments de géométrie hyperbolique). C'est en fait le théâtre de l'essentiel de l'action de $\Gamma$, et l'on s'y restreindra systématiquement. Si $\Gamma$ agit de façon cocompacte sur $X_{\Gamma}$, on dit que $\Gamma$ est convexe-cocompact; comme ces groupes n'offriront aucun intérêt dans une étude portant sur certaines compactifications du quotient $X_{\Gamma} / \Gamma$, passé quelques généralités, ils seront tout bonnement exclus à partir du paragraphe 3 , pour plus de commodité.

Pour $(\xi, x, y) \in X^{3}$, nous noterons $\beta_{\xi}(x, y)=d(x, \xi)-d(y, \xi)$. Il est bien connu que cette fonction de trois variables s'étend par continuité sur $\bar{X} \times X^{2}$. Restreinte à $\partial X \times X^{2}$, elle est connue sous le nom de fonction de Busemann. Mais nous aurons profit à considérer le cocycle de Busemann $\beta_{\xi}(x, y)$ défini pour 
$\xi$ dans $\bar{X}$ tout entier comme ci-dessus. Notons l'inégalité (d'origine triangulaire) $\left|\beta_{\xi}(x, y)\right| \leq d(x, y)$. Une horosphère dans $X$ est une «ligne de niveau » d'une fonction de Busemann $\beta_{\xi}(x,$.$) . Plus précisément, l'horosphère basée en \xi \in \partial X$ et passant par $x \in X$ est l'ensemble des $y \in X$ tels que $\beta_{\xi}(x, y)=0$.

2.2. Densités conformes invariantes. - On appellera densité une application $\mu$ qui à chaque $x \in X$ associe une mesure $\mu_{x}$ positive finie sur $\bar{X}$ (ordinairement sur $\partial X$, mais nous aurons avantage à considérer ici $\bar{X}$ tout entier). Une densité $\mu$ sera dite conforme de dimension $\delta \geq 0$ si pour tous $x$ et $x^{\prime}$ dans $X$, la mesure $\mu_{x^{\prime}}$ est absolument continue par rapport à $\mu_{x}$, avec une dérivée de Radon-Nikodym donnée par la formule :

$$
\frac{\mathrm{d} \mu_{x^{\prime}}}{\mathrm{d} \mu_{x}}(\xi)=e^{-\delta \beta_{\xi}\left(x^{\prime}, x\right)} .
$$

Une densité $\mu$ sera dite invariante par $\Gamma$ si pour tout $\gamma \in \Gamma$ et pour tout $x \in X$, on a $^{(1)}$ :

$$
\gamma_{*} \mu_{x}=\mu_{\gamma x} .
$$

Remarquons que le support fermé d'une densité $\mu$ conforme et invariante par $\Gamma$ (c'est-à-dire le support commun aux $\mu_{x}$ ) contient nécessairement $\Lambda(\Gamma)$. Nous désignerons désormais par $\mathscr{C}(\Gamma, \delta)$ (resp. $\mathscr{C}_{\infty}(\Gamma, \delta)$ ) l'ensemble des densités conformes de dimension $\delta$, invariantes par $\Gamma$, normalisées par $\left\|\mu_{o}\right\|=1$ où $o$ est un point dorénavant fixé dans $X_{\Gamma}$, et enfin portées par $\overline{X_{\Gamma}}(\operatorname{resp} . \Lambda(\Gamma)$ ).

L'exposant critique $\delta(\Gamma)$ de $\Gamma$ est par définition l'exposant critique de la série de type Dirichlet-Poincaré $G^{\delta}(x, y)=\sum_{\gamma \in \Gamma} e^{-\delta d(x, \gamma y)}(\delta \in \mathbb{R}$, le pourquoi de la notation $G$ apparaitra plus loin), ou encore $\delta(\Gamma)=\limsup _{t \rightarrow+\infty} \frac{1}{t} \log \sharp\{\gamma \in$ $\Gamma \mid d(x, \gamma y) \leq t\}$ (l'exposant ne dépend point du choix de $x$ et $y$ dans $X$ ). En réalité, la limite supérieure précédente est une véritable limite (cf [18]). Nous ferons désormais l'hypothèse que $\delta(\Gamma)$ est fini, hypothèse que l'étendue de notre cadre géométrique nous contraint de stipuler, mais qui est d'emblée acquise notamment lorsque $X$ est une variété à courbure pincée, ou encore lorsque $X$ admet un groupe d'isométries discret cocompact.

Patterson a montré que $\mathscr{C}_{\infty}(\Gamma, \delta(\Gamma))$ n'est pas vide, grâce à une célèbre construction (cf [17]). Notons au passage que cela fait voir que $\delta(\Gamma)>0$ (puisqu'il n'existe pas de mesure finie invariante par $\Gamma$ ). Sullivan a ensuite établi que $\mathscr{C}_{\infty}(\Gamma, \delta)$ est vide lorsque $\delta<\delta(\Gamma)$ (cf [21], [19]).

Quant aux densités dans $\mathscr{C}(\Gamma, \delta)$ portées par $X_{\Gamma}$, il est clair qu'il en existe si et seulement si $G^{\delta}(x, y)<\infty$ (ce qui ne dépend pas du choix de $x$ et $y$ que nous omettrons dorénavant dans cette condition), auquel cas ce sont les densités $\mathscr{G}^{\delta, y}=G(o, y)^{-1} \tilde{\mathscr{G}}^{\delta, y}\left(y \in X_{\Gamma}\right)$ et toutes leurs combinaisons selon des

(1) La mesure $\gamma_{*} \mu_{x}$ est définie par $\gamma_{*} \mu_{x}(B)=\mu_{x}\left(\gamma^{-1} B\right)$ pour tout borélien $B$

TOME $139-2011-\mathrm{N}^{\mathrm{O}} 1$ 
mesures positives finies portées par un domaine fondamental pour $\Gamma$ dans $X_{\Gamma}$, où $\tilde{\mathscr{G}}^{\delta, y}$ désigne la densité (non normalisée) donnée par

$$
\tilde{\mathscr{G}}_{x}^{\delta, y}=\sum_{\gamma \in \Gamma} e^{-\delta d(x, \gamma y)} \mathscr{D}_{\gamma y} \quad(x \in X) .^{(2)}
$$

2.3. Analogie avec les fonctions harmoniques et les potentiels. - Le fil conducteur de tout cet article est de considérer les densités de $\mathscr{C}_{\infty}(\Gamma, \delta)$ comme les fonctions harmoniques positives invariantes par $\Gamma$ de la théorie générale du potentiel; à proprement parler, les fonctions correspondantes sont données par les masses des densités : $x \mapsto\left\|\mu_{x}\right\|\left(\mu \in \mathscr{C}_{\infty}(\Gamma, \delta)\right)$, mais nous ne saurons qu'après le théorème 2 plus loin comment la correspondance entre densités et fonctions des masses peut être biunivoque. De même, toutes les densités de $\mathscr{C}(\Gamma, \delta)$ formeront pour nous la base (elles sont normalisées) du cône des fonctions surharmoniques positives, et les densités de $\mathscr{C}(\Gamma, \delta)$ portées par $X_{\Gamma}$ seront les potentiels. Pour tout ce qui a trait à la théorie du potentiel, nous renvoyons à [2].

Une fonction harmonique positive est dite minimale si toute autre fonction harmonique positive majorée par celle-là lui est proportionnelle; normalisées en un point $o$, elles forment l'ensemble des points extrémaux de la base du cône des harmoniques positives, et c'est ce qu'on appelle la frontière de Martin minimale. Ici, dans notre analogie, la frontière (de Martin) minimale sera l'ensemble $\mathcal{E}(\Gamma, \delta)$ des densités de $\mathscr{C}(\Gamma, \delta)$ ergodiques pour l'action de $\Gamma$; ce sont encore les points extrémaux de $\mathscr{C}_{\infty}(\Gamma, \delta)$. L'équivalence avec la définition en termes de fonctions sera discutée après le théorème 2 plus bas.

Lorsque $X$ est un espace hyperbolique de dimension finie $n$ (c'est-à-dire en courbure constante), $e^{\delta \beta_{\xi}(o, x)}$ est précisément le noyau de Poisson pour l'opérateur $\Delta+\delta(n-\delta)$ (où $\Delta$ désigne le laplacien), et les deux familles de densités coïncident. Mais dès que la courbure est variable, ces deux familles deviennent distinctes (voir à ce sujet [13]). Pourtant nous allons montrer que les densités conformes gardent bien des propriétés des harmoniques, même dans cette généralité, pourvu que l'espace $X_{\Gamma}$ jouisse d'une certaine uniformité que traduira le principe des ombres ci-dessous.

Le seul point trivial concerne les inégalités de Harnack; elles s'écrivent ici tout simplement :

$$
\forall \mu \in \mathscr{C}(\Gamma, \delta), x, y \in X, \quad e^{-\delta d(x, y)}\left\|\mu_{y}\right\| \leq\left\|\mu_{x}\right\| \leq e^{\delta d(x, y)}\left\|\mu_{y}\right\| .
$$

$\overline{\text { (2) Tout au long, }}, \mathscr{D}_{z}$ désignera la masse de Dirac au point $z$. 
2.4. Frontière de Martin. — Fixons $\delta$ tel que $G^{\delta}<\infty$. L'espace $X_{\Gamma} / \Gamma$ s'identifie à une partie de $\mathscr{C}(\Gamma, \delta)$ par le plongement $\Gamma y \mapsto \mathscr{G}^{\delta, y}$; cela amène une compactification métrisable ${\overline{X_{\Gamma} / \Gamma}}^{(\delta)}$ de $X_{\Gamma} / \Gamma$ dans $\mathscr{C}(\Gamma, \delta)$ (vu comme sous-espace de l'espace des probabilités sur $\Lambda(\Gamma)$ muni de la topologie faible- $*$ ), dont le bord $\left(\bar{X}_{\Gamma} / \Gamma^{(\delta)}\right) \backslash\left(X_{\Gamma} / \Gamma\right)$ sera noté $\mathcal{F}(\Gamma, \delta)$ et appelé frontière de Martin (pour la dimension $\delta$ ). Nous renvoyons toujours à [2] pour l'analogie avec la frontière de Martin classique (dont l'origine se trouve dans [15]). Nous dirons d'une densité $\zeta \in \mathcal{F}(\Gamma, \delta)$ qu'elle est un point de Martin; c'est une limite $\zeta=\lim _{n \rightarrow \infty} \mathscr{G}^{\delta, y_{n}}$ pour une suite $\Gamma y_{n}$ tendant vers l'infini (c'est-à-dire $d\left(o, \Gamma y_{n}\right) \rightarrow \infty$ ). Le noyau $(x, y) \mapsto \mathscr{G}_{x}^{\delta, y}$ est le noyau de Martin; par définition, il s'étend continûment sur le produit $X \times \bar{X}_{\Gamma} / \Gamma^{(\delta)}$. Nous verrons comment il est possible de retrouver le résultat fondamental qui affirme que la frontière de Martin contient la frontière minimale (théorème 4 plus bas), et par suite que le noyau de Martin permet de représenter toutes les (sur)harmoniques positives.

La proposition suivante résume la situation à ce stade; elle généralise un théorème de [22] obtenu en courbure constante par la théorie du potentiel liée au laplacien.

Proposition 1. - Si $\Gamma$ est convexe-cocompact, alors $\mathscr{C}_{\infty}(\Gamma, \delta)$ est un singleton si $\delta=\delta(\Gamma)$, et est vide si $\delta>\delta(\Gamma)$.

Si $\Gamma$ n'est pas convexe-cocompact, alors $\mathscr{C}_{\infty}(\Gamma, \delta)$ n'est pas vide si et seulement $\delta \geq \delta(\Gamma)$; si $G^{\delta}<\infty$, on a $\varnothing \varsubsetneqq \mathcal{F}(\Gamma, \delta) \subset \mathscr{C}_{\infty}(\Gamma, \delta)$.

Démonstration. - Le cas convexe-cocompact résulte de la construction de Patterson et du lemme de l'ombre de Sullivan, développé par un raisonnement de [21] (cf [19]).

Dans le cas où $X_{\Gamma} / \Gamma$ n'est pas compact, on voit par la discussion précédente que $\mathcal{F}(\Gamma, \delta) \neq \varnothing$ dès que $G^{\delta}<\infty$, notamment pour $\delta>\delta(\Gamma)$, d'où $\mathscr{C}_{\infty}(\Gamma, \delta) \neq$ $\varnothing$ pour $\delta>\delta(\Gamma)$, ce qui est encore vrai pour $\delta=\delta(\Gamma)$ par passage à la limite.

Nous supposerons désormais, comme annoncé au début, que le groupe $\Gamma$ n'est pas convexe-cocompact.

\section{Principe des ombres et théorème de Fatou}

La pierre angulaire de tout ce travail sera le principe énoncé ici, concernant les densités conformes d'une dimension donnée et les masses qu'elles donnent à certains sous-ensembles de $\bar{X}$, que nous allons à présent définir. Cette discussion reprend le point de départ de [20]. Pour $x, y \in X$ et $r>0$, nous appellerons ombre de la boule $B(y, r)$ dans $\bar{X}$ vue de $x$ l'ensemble (ouvert) $\emptyset_{r}(x, y)$ des 
points $\xi \in \bar{X}$ tels que le segment (ou rayon) géodésique partant de $x$ et finissant en $\xi$ rencontre $B(y, r)$.

3.1. Principe des ombres. - Observons d'abord que pour tout $\xi \in \theta_{r}(x, y)$, on a $d(x, y)-2 r<\beta_{\xi}(x, y) \leq d(x, y)$ (la seconde inégalité étant universelle). Aussi voit-on, en utilisant (1), que pour toute densité $\mu \in \mathscr{C}(\Gamma, \delta)$,

$$
e^{-\delta d(x, y)} \mu_{y}\left(\theta_{r}(x, y)\right) \leq \mu_{x}\left(\Theta_{r}(x, y)\right) \leq e^{2 \delta r} e^{-\delta d(x, y)} \mu_{y}\left(\theta_{r}(x, y)\right)
$$

En particulier, $\mu_{x}\left(\theta_{r}(x, y)\right) \leq e^{2 \delta r} e^{-\delta d(x, y)}\left\|\mu_{y}\right\|$; nous sommes à présent en mesure d'énoncer le principe suivant, qui pose l'inégalité inverse.

Nous dirons d'un sous-ensemble $Y \subset X_{\Gamma}$ invariant par $\Gamma$ qu'il vérifie le principe des ombres en $\delta \geq \delta(\Gamma)$ si pour toute densité $\mu \in \mathscr{C}(\Gamma, \delta)$, pour tous $x, y \in Y$, on a

$$
\frac{1}{C}\left\|\mu_{y}\right\| e^{-\delta d(x, y)} \leq \mu_{x}\left(\vartheta_{r}(x, y)\right) \leq C\left\|\mu_{y}\right\| e^{-\delta d(x, y)},
$$

pour un rayon $r=r(Y, \delta)$ et une constante $C=C(Y, \delta)$ indépendants de $x, y, \mu$. Nous dirons également que $Y$ vérifie le principe des ombres sur un intervalle $I$ s'il le vérifie en tout $\delta \in I$ avec $r(Y, I)=\sup _{\delta \in I} r(Y, \delta)<\infty$ et $C(Y, I)=\sup _{\delta \in I} C(Y, \delta)<\infty$.

Rappelons encore (voyez (5)) que l'inégalité de droite est universellement acquise avec pour constante $e^{2 \delta r}$, et que celle de gauche équivaut à $\mu_{y}\left(\theta_{r}(x, y)\right) \geq$ $\frac{1}{C^{\prime}}\left\|\mu_{y}\right\|$ avec une autre constante $C^{\prime}$.

Il est bien connu que l'orbite $\Gamma o$ vérifie le principe des ombres : c'est le lemme de l'ombre de Sullivan (voir [21], [19]). Mais le théorème suivant exhibe un ensemble généralement plus vaste satisfaisant aux axiomes requis. La possibilité de trouver, au moins dans des cas particuliers, d'autres tels ensembles, justifie qu'on ne se restreigne pas à la seule construction indiquée ici, mais que l'on développe la théorie avec un ensemble $Y$ général.

THÉORÈme 1. - Etant donné un sous-groupe non-élémentaire $\Gamma^{\prime} d e \Gamma$, soit $\mathcal{N}$ l'ensemble des isométries $\phi$ de $X$ telles que $\phi \Gamma^{\prime} \phi^{-1} \subset \Gamma$. Alors $Y=\mathcal{N a}$ vérifie le principe des ombres sur tout intervalle borné $\left[\delta(\Gamma), \delta_{0}\right]$. C'est encore vrai pour une réunion finie de tels ensembles.

Notons que l'ensemble $Y=\mathcal{N a}$ contient l'orbite de $o$ sous le normalisateur de $\Gamma$ dans le groupe des isométries de $X$.

Une situation intéressante s'offre à nous quand le groupe $\Gamma$ est distingué dans un autre groupe $\Gamma_{0}$ : alors $Y=\Gamma_{0} o$ vérifie le principe des ombres; si $\Gamma_{0}$ est convexe-cocompact, alors $X_{\Gamma}$ lui-même convient. 
Démonstration. - On notera ici $\tilde{\mathscr{C}}\left(\Gamma^{\prime}, \delta\right)$ l'ensemble des densités conformes de dimension $\delta$, invariantes par $\Gamma^{\prime}$, normalisées en $o$, mais dont le support fermé n'est pas nécessairement réduit à $X_{\Gamma}^{\prime}$ (il ne laisse cependant pas de contenir $\left.\Lambda\left(\Gamma^{\prime}\right)\right)$. On choisira $r>0$ assez grand pour que le complémentaire dans $\partial X$ de toute ombre de la boule $B(o, r)$ soit de diamètre plus petit que celui de $\Lambda\left(\Gamma^{\prime}\right)$ (pour une quelconque distance sur $\partial X$, par exemple la distance visuelle), lequel n'est pas nul du fait que $\Gamma^{\prime}$ n'est pas élémentaire; de sorte que toute ombre $\vartheta_{r}(x, o)(x \in \bar{X})$ rencontre $\Lambda\left(\Gamma^{\prime}\right)$.

Or on voit aisément que l'ensemble $K=\bar{X} \times \bigcup_{\delta \in\left[\delta(\Gamma), \delta_{0}\right]} \tilde{\mathscr{C}}\left(\Gamma^{\prime}, \delta\right)$ muni de la topologie usuelle est compact, et que l'application de $K$ dans $\mathbb{R}$ qui à $(x, \mu)$ associe $\mu_{o}\left(\theta_{r}(x, o)\right)$ est semi-continue inférieurement; elle admet donc un minimum, qui est nécessairement strictement positif, disons $\frac{1}{C}$, puisque le support fermé de $\mu_{o}$ contient $\Lambda\left(\Gamma^{\prime}\right)$ tout entier ( $\Gamma^{\prime}$ n'étant pas élémentaire), lequel rencontre chaque ouvert $\theta_{r}(x, o)$ de $\bar{X}$.

Considérons maintenant $\mu \in \mathscr{C}(\Gamma, \delta)$ avec $\delta \in\left[\delta(\Gamma), \delta_{0}\right]$, et $y=\phi o \in \mathcal{N} a=Y$. Soit $\nu_{x}=\left\|\mu_{\phi o}\right\|^{-1} \phi_{*}^{-1} \mu_{\phi x}(x \in X)$; il est facile de voir que $\nu$ est encore une densité conforme de dimension $\delta$; de plus, $\nu$ est invariante par $\Gamma^{\prime}$, puisque pour tout $\gamma \in \Gamma^{\prime}$, on a $\gamma_{*} \phi_{*}^{-1} \mu_{\phi x}=\phi_{*}^{-1}\left(\phi \gamma \phi^{-1}\right)_{*} \mu_{\phi x}=\phi_{*}^{-1} \mu_{\phi \gamma x}, \operatorname{car} \phi \gamma \phi^{-1} \in \Gamma$; enfin $\nu \in \tilde{\mathscr{C}}\left(\Gamma^{\prime}, \delta\right)$. Donc $\nu_{o}\left(\theta_{r}\left(\phi^{-1} x, o\right)\right) \geq \frac{1}{C}$ par ce qui précède, ce qui donne $\mu_{y}\left(\theta_{r}(x, y)\right) \geq \frac{1}{C}\left\|\mu_{y}\right\|$ comme voulu (voir la discussion plus haut).

3.2. Lemme à la Vitali. - Le principe des ombres sera souvent appliqué par l'intermédiaire d'une propriété combinatoire fondamentale des ombres autorisant des recouvrements de type Vitali : c'est le lemme suivant.

Lemme 1. - Soient $x \in X, r>0$ et $Z$ un sous-ensemble discret de $X$. Il existe $Z^{*} \subset Z$ tel que les ombres $\theta_{r}(x, y), y \in Z^{*}$ soient deux à deux disjointes, et $\bigcup_{y \in Z} \theta_{r}(x, y) \subset \bigcup_{y \in Z^{*}} \theta_{5 r}(x, y)$.

Démonstration. - Ecrivons $Z=\left\{z_{i}\right\}_{i \in \mathbb{N}}$ avec $d\left(x, z_{i}\right) \leq d\left(x, z_{i+1}\right)$ pour tout $i$. Définissons $i_{1}=0$, puis $i_{k+1}=\min \left\{i>i_{k} \mid \theta_{r}\left(x, z_{i}\right) \cap \bigcup_{j \leq k} \theta_{r}\left(x, z_{i_{j}}\right)=\varnothing\right\}$ par récurrence. Si $i_{k}<i<i_{k+1}$ pour un $k$, alors $\theta_{r}\left(x, z_{i}\right) \cap \theta_{r}\left(x, z_{i_{j}}\right) \neq$ $\varnothing$ pour un $j \leq k$, et l'on dispose d'un rayon géodésique partant de $x$ qui traverse $B\left(z_{i_{j}}, r\right)$ et $B\left(z_{i}, r\right)$; s'il les rencontre dans cet ordre, alors $\theta_{r}\left(x, z_{i}\right) \subset$ $\theta_{3 r}\left(x, z_{i_{j}}\right)$ par un simple argument de géométrie hyperbolique; sinon, on voit que $B\left(z_{i}, r\right) \subset B\left(z_{i_{j}}, 5 r\right)$ du fait que $d\left(x, z_{i}\right) \geq d\left(x, z_{i_{j}}\right)$; on en conclut dans tous les cas que $\theta_{r}\left(x, z_{i}\right) \subset \vartheta_{5 r}\left(x, z_{i_{j}}\right)$. Nous avons ainsi établi le lemme avec $Z^{*}=\left\{z_{i_{j}}\right\}_{j \in \mathbb{N}^{*}}$. 
3.3. Limite conique et théorème de Fatou. - Pour $Y \subset X_{\Gamma}$ invariant par $\Gamma$, nous aurons à considérer l'ensemble limite conique de $Y$, noté $\Lambda_{c}(Y)$, c'està-dire l'ensemble des points $\xi$ de $\partial X$ (en fait de $\Lambda(\Gamma)$ ) qui sont approchés coniquement par des points de $Y$, c'est-à-dire par des points de $Y$ restant à distance bornée d'un rayon géodésique finissant en $\xi$. En particulier, l'ensemble $\Lambda_{c}(\Gamma x)$, clairement indépendant du choix de $x \in X$ et noté plus simplement $\Lambda_{c}(\Gamma)$, est l'ensemble limite conique de $\Gamma$, étudié par exemple dans [19].

Pour mieux cerner l'approche conique des points de $\Lambda_{c}(Y)$, nous noterons, pour $r \in] 0, \infty], \Lambda_{c}^{r}(Y)$ l'ensemble des $\xi \in \partial X$ tels qu'il existe une suite de $y_{n} \in Y$ tendant vers $\xi$, avec $\xi \in \Theta_{\rho}\left(x, y_{n}\right)$ pour un $\rho$ strictement inférieur à $r$ (autrement dit la distance de $y_{n}$ au rayon $[o \xi)$ reste bornée par $\rho$ ). Ainsi définis, les ensembles $\Lambda_{c}^{r}(Y)$ sont invariants par $\Gamma$, et croissent avec $r$ vers $\Lambda_{c}^{\infty}(Y)=\Lambda_{c}(Y)$. Le lemme suivant nous sera utile.

Lemme 2. - Si $Y$ vérifie le principe des ombres en $\delta$, alors, dès que $r>$ $r(Y, \delta)$, l'ensemble $\Lambda_{c}(Y) \backslash \Lambda_{c}^{r}(Y)$ est négligeable pour toutes les densités de $\mathcal{C}(\Gamma, \delta)$.

Démonstration. - Fixons $r>\rho=r(Y, \delta)$. Considérons d'abord $\mu \in \mathcal{E}(\Gamma, \delta)$. Si $\mu_{o}\left(\Lambda_{c}(Y)\right)=0$, le résultat est clair. Sinon, $\mu_{o}\left(\Lambda_{c}(Y)\right)=1$ par ergodicité, puis $\mu_{o}\left(\Lambda_{c}^{R}(Y)\right)>0$ pour un $R>\rho$ assez grand, et donc $\mu_{o}\left(\Lambda_{c}^{R}(Y)\right)=1$. Le lemme 1 , appliqué avec $R$ à la place de $r$ et $x=o$, nous fournit $Z_{n}^{*}$ pour $Z_{n}=Y \backslash B(o, n)(n \in \mathbb{N})$. Désignons par $\Omega_{n}$ la réunion $\bigcup_{y \in Z_{n}^{*}} \vartheta_{\rho}(o, y)$, a fortiori disjointe (puisque $\rho<R$ ). En appliquant le principe des ombres, et en notant $C=\max \left(C(Y, \delta), e^{10 \delta R}\right)$, on obtient que $\mu_{o}\left(\Omega_{n}\right)=\sum_{y \in Z_{n}^{*}} \mu_{o}\left(\theta_{\rho}(o, y)\right) \geq \frac{1}{C} \sum_{y \in Z_{n}^{*}}\left\|\mu_{y}\right\| e^{-\delta d(o, y)} \geq$ $\frac{1}{C^{2}} \sum_{y \in Z_{n}^{*}} \mu_{o}\left(\theta_{5 R}(o, y)\right) \geq \frac{1}{C^{2}} \mu_{o}\left(\bigcup_{y \in Z_{n}} \theta_{R}(o, y)\right) \geq C^{-2} \mu_{o}\left(\Lambda_{c}^{R}(Y)\right)=C^{-2}$. Comme $\Lambda_{c}^{r}(Y)$ contient l'intersection décroissante des $\bigcup_{y \in Z_{n}} \theta_{\rho}(o, y) \supset \Omega_{n}$, on en déduit que $\mu_{o}\left(\Lambda_{c}^{r}(Y)\right) \geq C^{-2}>0$, et donc $\mu_{o}\left(\Lambda_{c}^{r}(Y)\right)=1$.

Nous avons ainsi montré que $\Lambda_{c}(Y) \backslash \Lambda_{c}^{r}(Y)$ est négligeable pour toutes les densités ergodiques de $\mathscr{C}(\Gamma, \delta)$, ce qu'on étend de là facilement à toutes les autres densités.

L'ensemble limite conique de $Y$ étant justement le lieu où le principe des ombres nous renseigne avec précision sur les densités conformes invariantes par $\Gamma$ à l'infini, par l'intermédiaire du lemme 1 , nous aboutissons au théorème suivant; à noter que les constantes inhérentes au principe des ombres s'effacent du moment qu'on se place à l'infini. Selon l'analogie présentée dans l'introduction, on retrouve ici le théorème de Fatou de la théorie du potentiel classique. Nous reviendrons plus loin sur ce théorème à propos de la notion d'effilement, en indiquant une seconde démonstration (toutefois un peu moins générale). 
ThÉORÈme 2. - ([20]) Supposons que $Y$ vérifie le principe des ombres en $\delta$. Considérons deux densités $\mu \in \mathscr{C}(\Gamma, \delta)$ et $\nu \in \mathscr{C}_{\infty}(\Gamma, \delta)$. Alors, pour $\nu_{o}$-presque tout $\xi \in \Lambda_{c}(Y)$, on a, lorsque $y \in Y$ tend vers $\xi$ coniquement,

$$
\frac{\left\|\mu_{y}\right\|}{\left\|\nu_{y}\right\|} \rightarrow \frac{\mathrm{d} \mu_{o}}{\mathrm{~d} \nu_{o}}(\xi) .
$$

Afin d'être complet, nous en reproduisons ici la démonstration.

Démonstration. - Etant donné $r \in] r(Y, \delta), \infty]$, définissons, pour tout $\xi \in$ $\Lambda_{c}^{r}(Y), D_{r}^{+}(\mu / \nu)(\xi)$ (resp. $\left.D_{r}^{-}(\mu / \nu)(\xi)\right)$ comme la limite croissante (resp. décroissante), lorsque $\rho<r$ croît vers $r$, de la limite supérieure (resp. inférieure) du rapport $\frac{\left\|\mu_{y}\right\|}{\left\|\nu_{y}\right\|}$ quand $y \in Y$ tend vers $\xi$ en restant à distance au plus $\rho$ du rayon $[o \xi)$. Observons que les fonctions $D_{r}^{+}(\mu / \nu)$ sont boréliennes et invariantes par $\Gamma$. La première étape traduira le principe des ombres; les suivantes développeront des arguments généraux pour achever la preuve. Fixons désormais $r \in] r(Y, \delta), \infty[$.

Première étape : Si $D_{r}^{+}(\mu / \nu) \geq 1$ sur $\Lambda_{c}^{r}(Y)$ modulo $\nu_{o}$, alors $\mu \geq \frac{1}{2 C^{2}} \nu$ sur $\Lambda_{c}^{r}(Y)$, où $C=\max \left(C(Y, \delta), e^{10 \delta r}\right)$.

Supposons que $D_{r}^{+}(\mu / \nu) \geq 1$ sur $\Lambda_{c}^{r}(Y)$ modulo $\nu_{o}$. Considérons un borélien $B \subset \bar{X}$, un ouvert $V \supset B$, puis l'ensemble $Z$ des $y \in Y$ tels que $\theta_{r}(o, y) \subset V$ et $\left\|\mu_{y}\right\| \geq \frac{1}{2}\left\|\nu_{y}\right\|$, auquel le lemme 1 associe $Z^{*}$. Comme $B \cap \Lambda_{c}^{r}(Y)$ est contenu modulo $\nu_{o}$ dans $\bigcup_{y \in Z} \theta_{r}(o, y)$, on obtient, en utilisant le principe des ombres, que $\nu_{o}\left(B \cap \Lambda_{c}^{r}(Y)\right) \leq \sum_{y \in Z^{*}} \nu_{o}\left(\theta_{5 r}(o, y)\right) \leq C \sum_{y \in Z^{*}}\left\|\nu_{y}\right\| e^{-\delta(o, y)} \leq$ $2 C \sum_{y \in Z^{*}}\left\|\mu_{y}\right\| e^{-\delta(o, y)} \leq 2 C^{2} \sum_{y \in Z^{*}} \mu_{o}\left(\theta_{r}(o, y)\right) \leq 2 C^{2} \mu_{o}(V)$. Comme $V$ était arbitraire, on en déduit que $\nu_{o}\left(B \cap \Lambda_{c}^{r}(Y)\right) \leq 2 C^{2} \mu_{o}(B)$, puis le résultat annoncé.

Seconde étape : Si $D_{r}^{+}(\mu / \nu) \geq 1$ sur $\Lambda_{c}^{r}(Y)$ modulo $\nu_{o}$, alors $\mu \geq \nu$ sur $\Lambda_{c}^{r}(Y)$.

Supposons que $D_{r}^{+}(\mu / \nu) \geq 1$ sur $\Lambda_{c}^{r}(Y)$ modulo $\nu_{o}$. Soit $\tau$ le plus grand réel tel que $\mu \geq \tau \nu$ sur $\Lambda_{c}^{r}(Y)$, et supposons par l'absurde que $\tau<1$. Soit $\mu^{\prime}=$ $(1-\tau)^{-1}\left(\mu-\tau \mathbb{I}_{\Lambda_{c}^{r}(Y)} \nu\right)$; on a bien $\mu^{\prime} \in \mathbb{R}^{+} \mathscr{C}(\Gamma, \delta)$. Or on vérifie immédiatement que $D_{r}^{+}\left(\mu^{\prime} / \nu\right) \geq 1$ sur $\Lambda_{c}^{r}(Y)$ modulo $\nu_{o}$, ce qui entraîne, par la première étape, que $\mu^{\prime} \geq \frac{1}{2 C^{2}} \nu$ sur $\Lambda_{c}^{r}(Y)$, ou encore $\mu \geq\left(\tau+\frac{1-\tau}{2 C^{2}}\right) \nu$ sur $\Lambda_{c}^{r}(Y)$, ce qui est contradictoire avec le choix de $\tau$.

Troisième étape : On a $D_{r}^{+}(\mu / \nu) \leq \frac{d \mu_{o}}{d \nu_{o}} \operatorname{sur} \Lambda_{c}^{r}(Y)$ modulo $\nu_{o}$.

Supposons par l'absurde que le sous-ensemble $A=\left\{D_{r}^{+}(\mu / \nu)>\epsilon+\frac{d \mu_{o}}{d \nu_{o}}\right\}$ de $\Lambda_{c}^{r}(Y)$ ne soit pas $\nu_{o}$-négligeable pour un $\epsilon>0$. Choisissons alors $s \geq 0$ tel que $\nu_{o}(A \cap B)>0$ où $B=\left\{s \leq \frac{d \mu_{o}}{d \nu_{o}}<s+\frac{\epsilon}{2}\right\}$. Considérons $\nu^{\prime}=\mathbb{I}_{A \cap B} \nu$ (A comme B sont invariants par $\Gamma)$. On a $D_{r}^{+}\left(\mu / \nu^{\prime}\right) \geq D_{r}^{+}(\mu / \nu) \geq \epsilon+s$ sur $A \cap B$, et donc modulo $\nu_{o}^{\prime}$. D'après la seconde étape, on en déduit que $\mu \geq(\epsilon+s) \nu^{\prime}$, et l'on aboutit à la contradiction que modulo $\nu_{o}$ sur $A \cap B$, on a $\frac{d \mu_{o}}{d \nu_{o}} \geq \epsilon+s>\frac{\epsilon}{2}+\frac{d \mu_{o}}{d \nu_{o}}$.

TOME $139-2011-\mathrm{N}^{\mathrm{O}} 1$ 
Quatrième étape : On a $D_{r}^{-}(\mu / \nu) \geq \frac{d \mu_{o}}{d \nu_{o}}$ sur $\Lambda_{c}^{r}(Y)$ modulo $\nu_{o}$.

Soit $\mu^{\prime}=\frac{d \mu_{o}}{d \nu_{o}} \nu$. Si $\mu^{\prime}=0$, il n'y a rien à montrer. Dans le cas contraire, on a $D_{r}^{-}(\mu / \nu) \geq D_{r}^{-}\left(\mu^{\prime} / \nu\right)=\left\{D_{r}^{+}\left(\nu / \mu^{\prime}\right)\right\}^{-1} \geq\left\{\frac{d \nu_{o}}{d \mu_{o}^{\prime}}\right\}^{-1}$ sur $\Lambda_{c}^{r}(Y)$ modulo $\mu_{o}^{\prime}$ grâce à la troisième étape. Or ce dernier terme vaut $\frac{d \mu_{o}}{d \nu_{o}}$ modulo $\mu_{o}^{\prime}$. Le résultat s'ensuit.

Conclusion : Il résulte des deux dernières étapes que $\frac{d \mu_{o}}{d \nu_{o}} \leq D_{r}^{-}(\mu / \nu) \leq$ $D_{r}^{+}(\mu / \nu) \leq \frac{d \mu_{o}}{d \nu_{o}}$ sur $\Lambda_{c}^{r}(Y)$ modulo $\nu_{o}$. En faisant tendre $r$ vers l'infini, il vient que $D_{\infty}^{ \pm}(\mu / \nu)=\frac{d \mu_{o}}{d \nu_{o}}$ sur $\Lambda_{c}(Y)$ modulo $\nu_{o}$, ce qui est exactement l'énoncé du théorème.

Le corollaire suivant nous permettra de poursuivre une discussion laissée ouverte dans l'introduction au sujet de l'analogie avec les fonctions harmoniques.

Corollaire 1. - (i) Supposons que $Y$ vérifie le principe des ombres en $\delta$ et $\delta^{\prime}$. Si les densités $\mu \in \mathscr{C}(\Gamma, \delta)$ et $\mu^{\prime} \in \mathscr{C}\left(\Gamma, \delta^{\prime}\right)$ sont portées par $\Lambda_{c}(Y)$, et si pour tout $y \in Y$ on a $\left\|\mu_{y}\right\|=\left\|\mu_{y}^{\prime}\right\|$, alors $\mu=\mu^{\prime}$ (en particulier $\left.\delta=\delta^{\prime}\right)$.

(ii) Supposons que $Y$ vérifie le principe des ombres en $\delta$. Soient $\mu \in \mathscr{C}(\Gamma, \delta)$ et $\mu^{\prime} \in \mathscr{C}(\Gamma, \delta)$. Si $\mu$ est portée par $\Lambda_{c}(Y)$, et si pour une constante $C>0$ et pour tout $y \in Y$ on a $\left\|\mu_{y}\right\| \leq C\left\|\mu_{y}^{\prime}\right\|$, alors $\mu \leq C \mu^{\prime}$.

Démonstration. - (i) Montrons d'abord l'égalité des exposants. On peut supposer que $\delta \geq \delta^{\prime}$. Prenons $R>0$ arbitrairement grand, et appliquons le lemme 1 avec $Z=Y \backslash B(o, R), x=o$ et $r>r(Y, \delta)$ fixé. En utilisant le lemme 2, le lemme 1 et le principe des ombres, on a $1=\mu_{o}\left(\Lambda_{c}^{r}(Y)\right) \leq \mu_{o}\left(\bigcup_{z \in Z^{*}} \theta_{5 r}(o, z)\right) \leq$ $e^{10 \delta r} \sum_{z \in Z^{*}}\left\|\mu_{z}\right\| e^{-\delta d(o, z)} \leq e^{-\left(\delta-\delta^{\prime}\right) R} e^{10 \delta r} \sum_{z \in Z^{*}}\left\|\mu_{z}^{\prime}\right\| e^{-\delta^{\prime} d(o, z)} \leq$ $e^{-\left(\delta-\delta^{\prime}\right) R} e^{10 \delta r} C(Y, \delta)$. Aussi a-t-on nécessairement que $\delta=\delta^{\prime}$. Le théorème 2 permet maintenant de conclure.

(ii) Soit $\epsilon>0$. Comme $\mu^{\prime}$ est absolument continue par rapport à $\mu+\epsilon \mu^{\prime}$, de dérivée de Radon-Nikodym disons $\rho$, et que $\frac{1}{C+\epsilon} \leq \frac{\left\|\mu_{y}^{\prime}\right\|}{\left\|\mu_{y}+\epsilon \mu_{y}^{\prime}\right\|} \leq \frac{1}{\epsilon}$, le théorème 2 montre que $\frac{1}{C+\epsilon} \leq \rho \leq \frac{1}{\epsilon}$ presque partout sur $\Lambda_{c}(Y)$, d'où il vient que $\mu=\mathbb{I}_{\Lambda_{c}(Y)} \frac{1-\epsilon \rho}{\rho} \mu^{\prime} \leq(C+\epsilon) \mu^{\prime}$. Comme $\epsilon$ est arbitrairement petit, le résultat cherché s'ensuit.

Si nous faisons l'hypothèse qu'il existe $Y$ vérifiant le principe des ombres en $\delta$ et tel que $\Lambda_{c}(Y)=\Lambda(\Gamma)$ (en particulier, si $X_{\Gamma}$ lui-même vérifie le principe des ombres), nous voyons à présent que, comme annoncé dans l'introduction, une densité conforme invariante est bien déterminée par la fonction donnée par ses masses, ce qui rend plus nette notre analogie avec les fonctions harmoniques. On voit notamment que la fonction « harmonique positive $x \mapsto\left\|\mu_{x}\right\|(\mu \in$ $\mathscr{C}_{\infty}(\Gamma, \delta)$ ) est minimale (c'est-à-dire que s'il existe $c>0$ telle que $\left\|\mu_{x}\right\| \geq c\left\|\nu_{x}\right\|$ 
$(x \in Y)$ pour une $\nu \in \mathscr{C}_{\infty}(\Gamma, \delta)$, alors $\left.\mu=\nu\right)$ si et seulement si $\mu \in \mathcal{E}(\Gamma, \delta)$. Nos définitions correspondent donc bien aux notions classiques.

\section{Réduite}

4.1. Théorème principal. - Dans ce paragraphe, nous mettons sur pied pour les densités conformes une opération fondamentale de la théorie du potentiel : celle de la réduite, d'où découlent bien d'autres notions essentielles, telles que l'effilement ou le balayage. Ce résultat s'obtient encore une fois à partir du principe des ombres et de considérations géométriques. Rappelons que dans notre analogie, les densités à l'infini (i.e. portées par $\partial X$ ) correspondent aux fonctions harmoniques, et que celles portées par $X$ correspondent aux potentiels. Il reste néanmoins cette différence avec la théorie classique que nos comparaisons sont dilatées par une constante $C$ (nous y reviendrons plus loin).

ThÉorème 3. - Soient $\delta$ tel que $G^{\delta}<\infty$, et $Y \subset X$ invariant par $\Gamma$ vérifiant le principe des ombres pour $\Gamma$ en $\delta$.

Pour toute densité $\mu \in \mathscr{C}(\Gamma, \delta)$ portée par $Y \cup \Lambda(\Gamma)$, pour tout $Z \subset Y$ invariant par $\Gamma$, il existe une densité $R_{Z} \mu \in \mathbb{R}^{+} \mathscr{C}(\Gamma, \delta)$ (non normalisée) portée par $Z \cup \Lambda_{c}(Z)$, telle que

$$
\forall z \in Z, \frac{1}{C}\left\|\mu_{z}\right\| \leq\left\|R_{Z} \mu_{z}\right\|, \quad \text { et } \quad \forall y \in Y,\left\|R_{Z} \mu_{y}\right\| \leq C\left\|\mu_{y}\right\|,
$$

où $C>0$ ne dépend que de $Y$ et $\delta$.

En outre, $R_{Z} \mu$ est linéaire en $\mu$ et crô̂t avec $\mu$; on a $R_{Z} \mu=\mu$ si $\mu$ est portée par $\Lambda_{c}(Z)$, et $R_{Z} \mu$ est portée par $Z$ si et seulement si $\mu_{o}\left(\Lambda_{c}(Z)\right)=0$.

Démonstration. - Prenons $r=4 r(Y, \delta)$ puis $R>r$ assez grand pour que $\sinh (R-r) \geq \frac{\sinh t}{\sinh (t+2 R-r)} \sinh (R+2 r)$ pour tout $t \geq 0$.

Commençons par discrétiser $Y$. Choisissons $Y^{\prime} \subset Y$ invariant par $\Gamma$ tel que $Y^{\prime} / \Gamma$ soit un sous ensemble $r$-séparé maximal de $Y / \Gamma$; soit alors $Z^{\prime}=\{y \in$ $\left.Y^{\prime} \mid B(y, r) \cap Z \neq \varnothing\right\}$. En vertu des inégalités de Harnack (4), il est clair qu'il suffit de montrer le théorème avec $Y^{\prime}$ et $Z^{\prime}$ à la place de $Y$ et $Z$ respectivement, quitte à modifier la constante $C$ obtenue. Aussi supposerons-nous désormais que $Y / \Gamma$ est $r$-séparé (on abandonnera donc la notation $Y^{\prime}$ ).

On se ramène ensuite au cas où $\mu_{o}\left(\Lambda_{c}(Z)\right)=0$, en posant $R_{Z} \mu=\mu$ sur $\Lambda_{c}(Z)$

Notons maintenant $\Xi=\partial^{2} X \cup(X \times \partial X)$ et $\pi: \Xi \rightarrow \bar{X}$ la première projection. Pour $z \in Z$, appelons $\phi_{z}^{0}$ l'ensemble des $(x, \eta) \in \Xi$ tels que le rayon géodésique ( $\eta x[$ parcouru vers $x$, après avoir rencontré $B(z, R)$, ne rencontre plus d'autre boule de rayon $R$ centrée en un point de $Z$. Soit enfin $\phi_{z}=\bigcup_{z^{\prime} \in \Gamma z \cap B(z, r)} \phi_{z^{\prime}}^{0}$ 
(en l'absence d'hypothèse de géométrie bornée sur $Y$, on ne peut se contenter de $\phi_{z}^{0}$ au lieu de $\left.\phi_{z}\right)$. Observons que $\gamma_{z}=\phi_{\gamma z}(\gamma \in \Gamma)$. On peut alors définir

$$
R_{Z} \mu_{x}=\sum_{z \in Z / \Gamma} \frac{\mu_{z}\left(\pi\left(\phi_{z}\right)\right)}{\sharp(\Gamma z \cap B(z, r))} \sum_{\gamma \in \Gamma} e^{-\delta d(x, \gamma z)} \mathscr{D}_{\gamma z} .
$$

C'est avec la réduite ainsi définie que nous allons montrer (8).

Après avoir fixé une quelconque densité auxiliaire $\nu \in \mathscr{C}_{\infty}(\Gamma, \delta)$ (il en existe par la proposition 1), introduisons la mesure $m$ sur $\Xi$ donnée par $\mathrm{d} m(x, \eta)=$ $e^{2 \delta\langle x, \eta\rangle_{y}} \mathrm{~d} \mu_{y}(x) \mathrm{d} \nu_{y}(\eta)$, où $\langle x, \eta\rangle_{y}=\frac{1}{2}\left[d(x, y)+\beta_{\eta}(y, x)\right]$ désigne le produit de Gromov, expression ici bien définie pour $x \in X$, mais qui s'étend continûment pour $x \in \bar{X}$ (voir [19],[6]). On vérifie sans peine que la formule définissant $m$ est bien indépendante du choix de $y \in X$, puis que $m$ est invariante par $\Gamma$. Pour plus de renseignements sur cette construction, nous renvoyons à [19].

Appelons $\left.\left.\mathscr{B}_{y, \rho}=\{(x, \eta) \in \Xi \mid] x \eta\right) \cap B(y, \rho) \neq \varnothing\right\}=\bigcup_{\eta \in \partial X} \theta_{\rho}(\eta, y) \times\{\eta\}$. On a immédiatement, grâce au principe des ombres, avec $C_{1}=e^{2 \delta r} C(Y, \delta)$ (cf (5) et (6)) :

$$
\begin{aligned}
& \forall y \in X, m\left(\mathscr{B}_{y, r}\right) \leq e^{\delta 2 r} \int_{\Lambda(\Gamma)} \mathrm{d} \nu_{y}(\eta) \mu_{y}\left(\vartheta_{r}(\eta, y)\right) \leq e^{\delta 4 r}\left\|\nu_{y}\right\|\left\|\mu_{y}\right\| \\
& \forall y \in Y, m\left(\mathscr{B}_{y, r}\right) \geq \int_{\Lambda(\Gamma)} \mathrm{d} \nu_{y}(\eta) \mu_{y}\left(\theta_{r}(\eta, y)\right) \geq \frac{1}{C_{1}}\left\|\nu_{y}\right\|\left\|\mu_{y}\right\|
\end{aligned}
$$

Prenons maintenant $z \in Z$ et montrons la première partie de (8). Comme $\bigcup_{y \in Z} \phi_{y}$ est l'ensemble des $(x, \eta) \in \Xi$ tels que le rayon géodésique ( $\eta x$ [ parcouru vers $x$ rencontre un nombre fini non-nul de boules de rayon $R>r$ centrées dans $Z$, on voit que $\mathcal{B}_{z, r} \backslash \pi^{-1}\left(\Lambda_{c}(Z)\right) \subset \bigcup_{y \in Z} \phi_{y}$ pour $z \in Z$. D'autre part, si $(x, \eta) \in \phi_{y}$, alors $(x, \eta) \in \phi_{\gamma_{0} y}^{0}$ pour un $\gamma_{0} y \in \Gamma y \cap B(y, r)$, puis $(x, \eta) \in$ $\bigcup_{y^{\prime} \in \Gamma y \cap B\left(\gamma_{0} y, r\right)} \phi_{y^{\prime}}$; le recouvrement $\left\{\phi_{y}\right\}_{y \in Z}$ a donc une multiplicité d'au moins $\sharp(\Gamma y \cap B(y, r))$ sur $\phi_{y}$. Puisque $\mu_{o}\left(\Lambda_{c}(Z)\right)=0$, on a par conséquent

$$
m\left(\mathscr{B}_{z, r}\right) \leq \sum_{y \in Z} \sharp(\Gamma y \cap B(y, r))^{-1} m\left(\phi_{y} \cap \mathcal{B}_{z, r}\right) .
$$

Or $\phi_{y} \cap \mathscr{B}_{z, r} \subset \bigcup_{x \in \pi\left(\phi_{y}\right)}\{x\} \times \mathscr{\theta}_{r}(x, z)$. Et si $(x, \eta) \in \phi_{y} \cap \mathscr{B}_{z, r}$, alors $(x, \eta) \in \phi_{y_{0}}^{0} \cap \mathscr{B}_{z, r}$ pour un $y_{0} \in \Gamma y \cap B(y, r)$, d'où $\langle x, \eta\rangle_{y}<\langle x, \eta\rangle_{y_{0}}+r<$ $R+r<2 R$ et $\beta_{\eta}(y, z) \geq \beta_{\eta}\left(y_{0}, z\right)-r \geq d\left(y_{0}, z\right)-4 R-r \geq d(y, z)-6 R$, 
puisque $] x \eta)$ rencontre d'abord $B(z, R)$ puis $B\left(y_{0}, R\right)$. Ainsi

$$
\begin{aligned}
\text { (conformité de } \nu) & \leq e^{10 \delta R} \int_{\pi\left(\phi_{y}\right)} \mathrm{d} \mu_{y}(x) \nu_{z}\left(\theta_{r}(x, z)\right) e^{-\delta d(y, z)} \\
& \leq e^{10 \delta R}\left\|\nu_{z}\right\| \mu_{y}\left(\pi\left(\phi_{y}\right)\right) e^{-\delta d(z, y)} .
\end{aligned}
$$

Avec (11) et (12), cela nous donne

$$
\left\|\nu_{z}\right\|\left\|\mu_{z}\right\| \leq C_{1} m\left(\mathscr{B}_{z, r}\right) \leq e^{10 \delta R} C_{1}\left\|\nu_{z}\right\| \sum_{y \in Z} \frac{\mu_{y}\left(\pi\left(\phi_{y}\right)\right)}{\sharp(\Gamma y \cap B(y, r))} e^{-\delta d(z, y)} .
$$

A l'aide d'un regroupement modulo $\Gamma$, au vu des diverses invariances par ce groupe, on obtient ainsi la première partie de (8), $R_{Z} \mu$ étant donnée par (9).

Considérons à présent, pour $z \in Z, \mathcal{J}_{z}=\bigcup_{x \in \pi\left(\phi_{z}\right)}\{x\} \times \Theta_{r}(x, z)$. On a clairement $\mathcal{J}_{\gamma z}=\gamma \mathcal{T}_{z}$ pour $\gamma \in \Gamma$.

Montrons d'abord que $d\left(z_{1}, z_{2}\right) \geq 2 R+r \Rightarrow \mathcal{J}_{z_{1}} \cap \mathcal{J}_{z_{2}}=\varnothing$, en supposant par l'absurde que $\mathcal{J}_{z_{1}} \cap \mathcal{J}_{z_{2}}$ contienne un point $(x, \eta)$ tandis que $d\left(z_{1}, z_{2}\right) \geq$ $2 R+r$. Le rayon $] x \eta)$ rencontre $B\left(z_{1}, r\right)$ et $B\left(z_{2}, r\right)$ en des points $z_{1}^{\prime}$ et $z_{2}^{\prime}$ respectivement, disons dans cet ordre en partant de $x$, quitte à échanger $z_{1}$ et $z_{2}$. Alors $d\left(z_{1}^{\prime}, z_{2}^{\prime}\right)>d\left(z_{1}, z_{2}\right)-2 r \geq 2 R-r$, d'où $d\left(x, z_{2}^{\prime}\right)>d\left(x, z_{1}^{\prime}\right)+2 R-r$. En appliquant le lemme géométrique rejeté à la fin de la démonstration en cours (lemme 3), avec pour données $x, z_{1}^{\prime}, z_{2}^{\prime}, R-r, R+2 r$ à la place de $x, y, z, r, R$, on obtient, compte tenu du choix de $R$ fait au début, que $B\left(z_{2}, R+r\right) \subset$ $\vartheta_{R+r}\left(x, z_{2}\right) \subset \vartheta_{R+2 r}\left(x, z_{2}^{\prime}\right) \subset \vartheta_{R-r}\left(x, z_{1}^{\prime}\right) \subset \vartheta_{R}\left(x, z_{1}\right)$. Mais $x \in \pi\left(\varnothing_{z_{2}}\right)$, et donc $x \in \pi\left(\phi_{z}^{0}\right)$ pour un certain $z \in Z \cap B\left(z_{2}, r\right)$, ce qui est contradictoire avec le fait que $B(z, R) \subset B\left(z_{2}, R+r\right) \subset \vartheta_{R}\left(x, z_{1}\right)$ et que $B\left(z_{1}, R\right) \cap B(z, R)=\varnothing$.

Il en résulte que le recouvrement $\left\{\mathcal{J}_{z}\right\}_{z \in Z}$ a une multiplicité bornée sur chaque $\mathcal{J}_{z}$ par $\sharp(Y \cap B(z, 2 R+r))$. D'après le lemme 4 , cette dernière quantité est bornée par $C_{2} \sharp(\Gamma z \cap B(z, r))$, où $C_{2}$ ne dépend ici plus que de $Y$ et $\delta$.

Prenons maintenant $y \in Y$ en vue d'établir la seconde partie de (8). Ce qui précède a montré que

$$
m\left(\mathcal{B}_{y, r}\right) \geq \frac{1}{C_{2}} \sum_{z \in Z} \sharp(\Gamma z \cap B(z, r))^{-1} m\left(\mathcal{J}_{z} \cap \mathcal{B}_{y, r}\right) .
$$

Or $m\left(\mathcal{J}_{z} \cap \mathcal{B}_{y, r}\right) \geq \mu_{z} \otimes \nu_{z}\left(\mathcal{J}_{z} \cap \mathcal{B}_{y, r}\right)=\int_{\pi\left(\phi_{z}\right)} \mathrm{d} \mu_{z}(x) \nu_{z}\left(\theta_{r}(x, z) \cap \theta_{r}(x, y)\right)$. En outre, $y \in \vartheta_{\frac{r}{2}}(x, z) \Rightarrow \vartheta_{\frac{r}{2}}(x, y) \subset \vartheta_{r}(x, z)$ par un simple argument géométrique, et $\nu_{z}\left(\Theta_{\frac{r}{2}}(x, y)\right) \geq e^{-\delta d(z, y)} \nu_{y}\left(\Theta_{\frac{r}{2}}(x, y)\right) \geq \frac{1}{C_{1}}\left\|\nu_{y}\right\| e^{-\delta d(z, y)}$ par le 
principe des ombres. Ainsi

$$
\begin{aligned}
m\left(\mathscr{B}_{y, r}\right) & \geq \frac{1}{C_{1} C_{2}}\left\|\nu_{y}\right\| \sum_{z \in Z} \sharp(\Gamma z \cap B(z, r))^{-1} \int_{\pi\left(\phi_{z}\right)} \mathrm{d} \mu_{z}(x) e^{-\delta d(z, y)} \mathbb{I}_{\emptyset_{\frac{r}{2}}(x, z)}(y) \\
& =\frac{1}{C_{1} C_{2}}\left\|\nu_{y}\right\| \sum_{z \in Z / \Gamma} \sharp(\Gamma z \cap B(z, r))^{-1} \int_{\pi\left(\phi_{z}\right)} \mathrm{d} \mu_{z}(x) \tilde{\mathscr{G}}_{z}^{\delta, y}\left(\oslash_{\frac{r}{2}}(x, z)\right)
\end{aligned}
$$

(où l'on a usé des diverses invariances par $\Gamma$; pour la notation, voyez (3)). Encore une fois grâce au principe des ombres, on a $\tilde{\mathscr{G}}_{z}^{\delta, y}\left(\Theta_{\frac{r}{2}}(x, z)\right) \geq$ $\frac{1}{C_{1}} \sum_{\gamma \in \Gamma} e^{-\delta d(z, \gamma y)}$ pour $x \in Y \cup \Lambda(\Gamma)$, donc $\mu_{z}$-presque partout. D'où $m\left(\mathcal{B}_{y, r}\right) \geq \frac{1}{C_{1}^{2} C_{2}}\left\|\nu_{y}\right\|\left\|R_{Z} \mu_{y}\right\|$, ce qui avec (10) termine la démonstration de (8), moyennant celle des deux lemmes ci-dessous.

Lemme 3. - Soient $x \in X, y, z \in X$ tels que $y$ soit sur le segment géodésique $] x z\left[\right.$. Si $r, R>0$ sont tels que $\sinh r \geq \frac{\sinh d(x, y)}{\sinh d(x, z)} \sinh R$, alors $\theta_{r}(x, y) \supset$ $\vartheta_{R}(x, z)$.

Démonstration. - Nous allons montrer qu'un point $a \in B(z, R)$ fixé appartient à $\vartheta_{r}(x, y)$. Pour ce faire, considérons un triangle de comparaison $\bar{a} \bar{x} \bar{z}$ dans $\mathbb{H}^{2}$ pour le triangle $a x z$. Appelons $p$ le projeté orthogonal sur la géodésique $(\bar{x} \bar{a})$ de l'image $\bar{y}$ de $y$ par cette comparaison.

Premier cas : $p \in[\bar{x} \bar{a}]$. Alors $p=\bar{b}$ pour un $b \in[x a]$. Notons $\theta$ la mesure de l'angle $\angle \bar{a} \bar{x} \bar{z}$. La loi des sinus (cf [5]) nous donne $\frac{\sin \theta}{\sinh d_{\mathbb{H}^{2}}(\bar{y}, \bar{b})}=$ $\frac{1}{\sinh d(x, y)}$ et $\frac{\sin \theta}{\sinh d(z, a)} \leq \frac{1}{\sinh d(z, x)}$, d'où $\sinh d(y, b) \leq \sinh d_{\mathbb{H}^{2}}(\bar{y}, \bar{b}) \leq$ $\frac{\sinh d(x, y)}{\sinh d(x, z)} \sinh d(x, a)<\sinh r$. Ainsi $a \in[x b) \subset \vartheta_{r}(x, y)$.

Second cas : $p \notin(\bar{x} \bar{a}]$. Notons $\alpha$ et $\beta$ les mesures des angles $\angle \bar{x} \bar{a} \bar{y}$ et $\angle \bar{x} \bar{a} \bar{z}$ respectivement. Puisque $\frac{\pi}{2} \leq \alpha \leq \beta \leq \pi$, on a $\sin \alpha \geq \sin \beta$. D'autre part, toujours par la loi des sinus, $\frac{\sin \theta}{\sinh d_{\mathbb{H}^{2}}(\bar{a}, \bar{y})}=\frac{\sin \alpha}{\sinh d(x, y)}$ et $\frac{\sin \theta}{\sinh d(z, a)}=\frac{\sin \beta}{\sinh d(z, x)}$, d'où $\sinh d(y, a) \leq \sinh d_{\mathbb{H}^{2}}(\bar{y}, \bar{a}) \leq \frac{\sinh d(x, y)}{\sinh d(x, z)} \sinh d(x, a)<\sinh r$. Ainsi $a \in$ $B(y, r)$.

Troisième cas : $p \notin[\bar{x} \bar{a})$. Alors $\theta>\frac{\pi}{2}$, d'où $d(x, a)>d(x, z)$, puis $\sinh d(x, y)<\frac{\sinh d(x, y)}{\sinh d(x, z)} \sinh d(x, a)<\sinh r$. En ce cas, $\theta_{r}(x, y)=X$.

Le lemme restant montre comment le principe des ombres impose une certaine uniformité géométrique à un ensemble $Y$ satisfaisant au principe des ombres, et en particulier à la fonction orbitale de $\Gamma$. Il nous fait l'économie d'une hypothèse de géométrie bornée sur $Y$, et nous sera de nouveau utile plus loin. 
Lemme 4. - Supposons que $Y \subset X$ invariant par $\Gamma$ vérifie le principe des ombres pour $\Gamma$ en $\delta \geq \delta(\Gamma)$ et que $Y / \Gamma$ est $r$-séparé, où $r=4 r(Y, \delta)$. Alors pour tout $R \geq r$, pour tout $y \in Y, \sharp(Y \cap B(y, R)) \leq M \sharp(\Gamma y \cap B(y, r))$ où $M$ ne dépend pas de $y$.

Démonstration. - Construisons d'abord une mesure $V$ sur $X_{\Gamma}$ comme suit; pour plus de détails, nous renvoyons à [19]. Commençons par choisir une densité $\nu \in \mathscr{C}_{\infty}(\Gamma, \delta)$ (cf proposition 1). Appelons $S X$ l'espace des géodésiques paramétrées, et $p: S X \rightarrow X$ la projection canonique; en identifiant $S X$ à $(\partial X \times \partial X) \backslash\{$ diagonale $\} \times \mathbb{R}$, on définit une mesure $m$ sur $S X$ par $\mathrm{d} m(\xi, \eta, t)=$ $e^{2 \delta\langle\xi, \eta\rangle_{y}} \mathrm{~d} \nu_{y}(\xi) \mathrm{d} \nu_{y}(\eta) \mathrm{d} t$ (expression indépendante $\mathrm{du}$ choix de $y \in X$ ), puis $V=p_{*} m$. La mesure $V$ est invariante par l'action de $\Gamma$ sur $X$, comme l'est $m$ sur $S X$.

On voit facilement que $V(B(y, \rho)) \leq \rho e^{2 \delta \rho}\left\|\nu_{y}\right\|^{2}$ pour tous $y \in X$ et $\rho>0$, et que si $y \in Y$ et $\rho \geq 2 r(Y, \delta)$, grâce au principe des ombres, $V(B(y, \rho)) \geq$ $\frac{\rho}{2} \int_{\Lambda(\Gamma)} \mathrm{d} \nu_{y}(\xi) \nu_{y}\left(\Theta_{\frac{\rho}{2}}(\xi, y)\right) \geq \frac{\rho e^{-\delta \rho}}{2 C(Y, \delta)}\left\|\nu_{y}\right\|^{2}$.

Montrons à présent le lemme en utilisant la mesure $V$ construite ci-dessus. Soient $R \geq r$ et $y \in Y$. Prenons $S \subset Y \cap B(y, R)$ un sous-ensemble $r$-séparé maximal. Alors $Y \cap B(y, R) \subset \bigcup_{x \in S} \Gamma x \cap B(x, r)$ (on rappelle que $Y / \Gamma$ est $r$-séparé), d'où

$$
\sharp(Y \cap B(y, R)) \leq(\sharp S) \max _{x \in S} \sharp(\Gamma x \cap B(x, r)) \leq(\sharp S) \sharp(\Gamma y \cap B(y, r+2 R)) .
$$

D'autre part, $B(y, R+r)$ contient la réunion disjointe des $B\left(x, \frac{r}{2}\right)(x \in S)$, d'où $\sum_{x \in S} V\left(B\left(x, \frac{r}{2}\right)\right) \leq V(B(y, R+r))$. Avec les estimées de la masse des boules pour $V$ ci-dessus, jointes aux inégalités de Harnack (4) pour $\nu$, on voit que $\sharp S \leq M_{1}(R)$ où $M_{1}(R)$ ne dépend, en dehors de $R$, que de $\delta, r$, et $C(Y, \delta)$.

En appliquant la première inégalité de (14) avec $\Gamma y$ au lieu de $Y$ et $r+2 R$ à la place de $R$, on obtient donc $\sharp(\Gamma y \cap B(y, r+2 R)) \leq M_{1}(r+2 R) \sharp(\Gamma y \cap B(y, r))$.

En reportant cette dernière inégalité dans (14), on aboutit au résultat voulu, avec $M=M_{1}(R) M_{1}(r+2 R)$.

La démonstration du théorème 3 est maintenant complète.

4.2. Effilement et théorème de Fatou. - Munis d'une telle notion de réduite, nous pouvons paraphraser une définition classique de la théorie du potentiel (cf [2]). Nous supposerons ici que $G^{\delta}<\infty$ et que $Y \subset X_{\Gamma}$ invariant par $\Gamma$ vérifie le principe des ombres pour $\Gamma$ en $\delta$, et tel que $\Lambda_{c}(Y)=\Lambda(\Gamma)$. Etant donné $\mu \in \mathcal{E}(\Gamma, \delta)$, nous dirons qu'un ensemble $Z \subset Y$ invariant par $\Gamma$ est $\mu$-effilé si $R_{Z} \mu \neq \mu$. L'alternative de Brelot a bien lieu : ou bien $R_{Z} \mu=\mu$, ou bien $R_{Z} \mu$ est un potentiel, c'est-à-dire que $R_{Z} \mu$ est portée par $Z$; en fait, $Z$ est $\mu$-effilé si et seulement si $\mu_{o}\left(\Lambda_{c}(Z)\right)=0$ (voyez le théorème 3 ). Nous dirons 
aussi qu'une fonction de $Y / \Gamma$ dans $\mathbb{R}$ admet une limite fine $l$ en $\lambda \in \mathcal{E}(\Gamma, \delta)$ si elle tend vers $l$ le long du filtre des parties de $Y / \Gamma$ de complémentaire $\lambda$-effilé. Des arguments classiques de la théorie du potentiel permettent alors d'établir le théorème de Fatou abstrait suivant (théorème de Fatou-Doob-Naim); pour $\mu \in \mathscr{C}(\Gamma, \delta)$, nous écrirons $\mu=\int_{\mathscr{E}(\Gamma, \delta)} \lambda \mathrm{d} \tilde{\mu}(\lambda)$ sa décomposition ergodique, ou représentation intégrale de Choquet (selon [8]).

ThÉORÈme. - Soient $\mu \in \mathscr{C}(\Gamma, \delta)$ et $\nu \in \mathscr{C}_{\infty}(\Gamma, \delta)$. Alors $\frac{\left\|\mu_{y}\right\|}{\left\|\nu_{y}\right\|}$ tend finement vers $\frac{\mathrm{d} \tilde{\mu}}{\mathrm{d} \tilde{\nu}}(\lambda)$ pour $\tilde{\nu}$-presque tout $\lambda \in \mathcal{E}(\Gamma, \delta)$.

Un peu de réflexion montre qu'il s'agit en fait du même théorème que celui déjà présenté sous ce nom, mais dont la forme paraît plus concrète (théorème 2). Voyons par exemple comment celui-là entraîne celui-ci.

Démonstration. - Envisageons d'abord le cas où $\mu$ et $\nu$ sont mutuellement singulières; il en est alors de même de $\tilde{\mu}$ et de $\tilde{\nu}$. Etant donné $\epsilon>0$, considérons l'ensemble $Z$ des $y \in Y$ tels que $\frac{\left\|\mu_{y}\right\|}{\left\|\nu_{y}\right\|} \geq \epsilon$. Alors $\int_{\mathscr{E}(\Gamma, \delta)} \lambda_{o}\left(\Lambda_{c}(Z)\right) \mathrm{d} \tilde{\nu}(\lambda)=$ $\nu_{o}\left(\Lambda_{c}(Z)\right)=0$ par le théorème 2 , d'où il ressort que $Z$ est $\lambda$-effilé $\tilde{\nu}$-presque partout, ce qui donne la conclusion voulue, $\epsilon$ étant arbitrairement petit.

Traitons maintenant le cas où $\mu=f \nu$; la dérivée de Radon-Nikodym $f$ est mesurable pour la tribu des invariants par $\Gamma$. Il suffit de traiter le cas où $f$ est proportionnelle à une fonction indicatrice, i.e. $f=\nu_{o}(A)^{-1} \mathbb{I}_{A}$ où $A$ est invariant par $\Gamma$; de là on passe au cas d'une fonction étagée, puis au cas général. Notons $\tilde{A}=\left\{\lambda \in \mathcal{E}(\Gamma, \delta) \mid \lambda_{o}(A)=1\right\}$, puis $\tilde{f}=\nu_{o}(A)^{-1} \mathbb{I}_{\tilde{A}} ;$ comme $\int_{\delta(\Gamma, \delta)} \lambda \mathrm{d} \tilde{\mu}(\lambda)=\mu=f \nu=\int_{\mathscr{E}(\Gamma, \delta)}(f \lambda) \mathrm{d} \tilde{\nu}(\lambda)=\int_{\mathscr{E}(\Gamma, \delta)} \lambda \tilde{f}(\lambda) \mathrm{d} \tilde{\nu}(\lambda)$, on a, par unicité de cette représentation intégrale, $\tilde{\mu}=\tilde{f} \tilde{\nu}$.

Cela dit, étant donné $\epsilon>0$, considérons l'ensemble $Z_{0}$ (resp. $Z_{1}$ ) des $y \in Y$ tels que $\frac{\left\|\mu_{y}\right\|}{\left\|\nu_{y}\right\|} \geq \epsilon$ (resp. $\left.\left|\frac{\left\|\mu_{y}\right\|}{\left\|\nu_{y}\right\|}-\nu_{o}(A)^{-1}\right| \geq \epsilon\right)$. Par le théorème 2, on a respectivement $\int_{\mathscr{E}(\Gamma, \delta)} \mathbb{I}_{\tilde{A}^{c}}(\lambda) \lambda_{o}\left(\Lambda_{c}\left(Z_{0}\right)\right) \mathrm{d} \tilde{\nu}(\lambda)=\int_{\mathscr{E}(\Gamma, \delta)} \lambda_{o}\left(A^{c} \cap \Lambda_{c}\left(Z_{0}\right)\right) \mathrm{d} \tilde{\nu}(\lambda)=$ $\nu_{o}\left(A^{c} \cap \Lambda_{c}\left(Z_{0}\right)\right)=0$ et $\int_{\tilde{E}(\Gamma, \delta)} \mathbb{I}_{\tilde{A}}(\lambda) \lambda_{o}\left(\Lambda_{c}\left(Z_{1}\right)\right) \mathrm{d} \tilde{\nu}(\lambda)=\nu_{o}\left(A \cap \Lambda_{c}\left(Z_{1}\right)\right)=0$, d'où vient que $Z_{0}$ est effilé $\tilde{\nu}$-presque partout sur $\tilde{A}^{c}$, et que $Z_{1}$ est effilé $\tilde{\nu}$ presque partout sur $\tilde{A}$; la conclusion voulue s'ensuit, $\epsilon$ étant arbitrairement petit.

4.3. Frontière de Martin et frontière minimale. - Grâce à notre construction de la réduite d'une densité conforme, nous sommes à présent en mesure de montrer que les densités conformes ergodiques sont des points de Martin comme nous les avons définis plus haut, pourvu que l'ensemble $Y$ du principe des ombres remplisse assez l'espace $X_{\Gamma}$. Dans le langage de la théorie de Martin, c'est exactement dire que la frontière de Martin contient la frontière minimale. On 
peut voir facilement que $\mathcal{E}(\Gamma, \delta)$ est un $G_{\delta}$ de $\mathcal{F}(\Gamma, \delta)$; les deux frontières restent en général distinctes.

ThÉORÈme 4. - Supposons que $X_{\Gamma}$ vérifie le principe des ombres en $\delta$ et que $G^{\delta}<\infty$. Alors toute densité de $\mathscr{C}_{\infty}(\Gamma, \delta)$ est limite (au sens de la topologie faible-*) d'une suite de densités de $\mathscr{C}(\Gamma, \delta)$ portées par $X_{\Gamma}$ (et même à support compact dans $X_{\Gamma}$ modulo $\left.\Gamma\right)$.

En outre, $\mathcal{E}(\Gamma, \delta) \subset \mathcal{F}(\Gamma, \delta):$ si $\mu \in \mathcal{E}(\Gamma, \delta)$, il existe une suite $\Gamma y_{n}$ dans $X_{\Gamma} / \Gamma$ tendant vers l'infini telle que $\mu=\lim _{n \rightarrow \infty} \mathscr{G}^{\delta, y_{n}}$.

$\mathrm{Si}$, par souci d'alléger, nous avons ici adopté l'hypothèse que $X_{\Gamma}$ vérifie luimême le principe des ombres, il ressort néanmoins de la démonstration que nous aurions aussi bien pu la remplacer par celle plus générale qu'il existe $Y \subset X$, invariant par $\Gamma$, vérifiant le principe des ombres pour $\Gamma$ en $\delta$, et tel que $\forall \mu \in \mathcal{E}(\Gamma, \delta), \mu_{o}\left(\Lambda_{c}(Y)\right)=1$ (ce qui est notamment le cas lorsque $\left.\Lambda_{c}(Y)=\Lambda(\Gamma)\right)$.

Démonstration. - Il suffit d'envisager le cas où $\mu$ est ergodique pour $\Gamma$; le reste en découle par combinaison convexe.

Soit $\left\{\Gamma K_{n}\right\}$ une exhaustion croissante de $X_{\Gamma}$ où chaque $K_{n}$ est compact. Comme $\Lambda_{c}\left(\Gamma K_{n}\right)=\Lambda_{c}(\Gamma)$ et que $\mu_{o}\left(\Lambda_{c}(\Gamma)\right)=0$ du fait que $\Gamma$ est convergent en $\delta$ (cf [19], th. 1.7), les densités $R_{\Gamma K_{n}} \mu$ sont portées par $\Gamma K_{n} \subset X_{\Gamma}$. Considérons une limite faible $\mu^{*}$ d'une sous-suite de $\left\{R_{\Gamma K_{n}} \mu\right\}$; on a alors $\frac{1}{C}\left\|\mu_{y}\right\| \leq\left\|\mu_{y}^{*}\right\| \leq$ $C\left\|\mu_{y}\right\|$ pour tout $y \in X_{\Gamma}$, où $C$ est la constante du théorème 3. Grâce au corollaire $1 \mathrm{du}$ théorème 2 à la Fatou, il s'ensuit que $\frac{1}{C} \leq \frac{\mathrm{d} \mu_{o}^{*}}{\mathrm{~d} \mu_{o}} \leq C$ ( $\mu_{o}$-p.p). L'ergodicité de $\mu$ pour $\Gamma$ montre alors que la dérivée $\frac{\mathrm{d} \mu_{o}^{*}}{\mathrm{~d} \mu_{o}}$, qui est essentiellement invariante par $\Gamma$, est essentiellement constante, et donc que $\mu^{*}=k \mu$ avec $\frac{1}{C} \leq k \leq C$.

Nous voyons ainsi apparaître $\mu$ comme combinaison de noyaux de Martin sur la frontière :

$$
\mu=\int_{\mathcal{F}(\Gamma, \delta)} \zeta \mathrm{d} \lambda(\zeta)
$$

où $\lambda$ est une mesure de probabilité (du fait de la normalisation en $o$ des densités). L'ergodicité de $\mu$ entraîne enfin que $\lambda$ est nécessairement une masse de Dirac.

Le théorème de Martin (cf [2]) ajoute encore que le complémentaire dans $X_{\Gamma} / \Gamma$ de tout voisinage d'un point de $\mu \in \mathcal{E}(\Gamma, \delta)$ dans $\mathcal{F}(\Gamma, \delta) \cup X_{\Gamma} / \Gamma$ (vu comme compactification de $\left.X_{\Gamma} / \Gamma\right)$ est $\mu$-effilé, ce qui se traduit dans notre cadre sous la forme plus concrète ci-dessous. Ce résultat précise beaucoup la façon dont les densités ergodiques apparaissent comme points de Martin. 
THÉORÈme 5. - Supposons que $X_{\Gamma}$ vérifie le principe des ombres en $\delta$ et que $G^{\delta}<\infty$. Pour tout $\mu \in \mathcal{E}(\Gamma, \delta)$, pour $\mu_{o}$-presque tout point $\xi \in \Lambda(\Gamma)$, on $a$, lorsque $y \in X_{\Gamma}$ tend vers $\xi$ coniquement,

$$
\mathscr{G}^{\delta, y} \rightarrow \mu \text {. }
$$

(Même remarque qu'au théorème précédent concernant l'hypothèse.)

Démonstration. - Reprenons la démonstration du théorème 4 précédent, avec $\left\{\Gamma K_{n}\right\}$ une exhaustion croissante de $X_{\Gamma}$ où chaque $K_{n}$ est compact. Soit $E$ l'intersection de $X_{\Gamma}$ et (du relèvement) du complémentaire d'un voisinage ouvert $V$ de $\mu \in \mathcal{E}(\Gamma, \delta)$ dans $\mathcal{F}(\Gamma, \delta) \cup X_{\Gamma} / \Gamma$ vu comme compactification de $X_{\Gamma} / \Gamma$. Supposons par l'absurde que $E$ ne soit pas $\mu$-effilé, autrement dit que $\mu_{o}\left(\Lambda_{c}(E)\right)=$ 1 (c'est notre caractérisation de l'effilement). Comme dans la démonstration précédente, soit $\mu^{*}$ une limite faible d'une sous-suite de $\left\{R_{E \cap \Gamma K_{n}} \mu\right\}$. Alors pour tout $y \in E\left(\operatorname{resp} . y \in X_{\Gamma}\right)$, on a $\left\|\mu_{y}\right\| \leq C\left\|\mu_{y}^{*}\right\|\left(\right.$ resp. $\left.\left\|\mu_{y}^{*}\right\| \leq C\left\|\mu_{y}\right\|\right)$, où $C$ est la constante du théorème 3 . Par le corollaire 1 appliqué avec $E$ (resp. $X_{\Gamma}$ ) à la place de $Y$, on en déduit que $\mu \leq C \mu^{*}\left(\right.$ vu que $\left.\mu_{o}\left(\Lambda_{c}(E)\right)=1\right)$ et que $\mathbb{I}_{\Lambda(\Gamma)} \mu^{*} \leq C \mu$. L'ergodicité de $\mu$ entraîne alors que $\mu=k \mathbb{I}_{\Lambda(\Gamma)} \mu^{*}$ où $k>0$. Ainsi $\mu=\int_{\mathscr{F}(\Gamma, \delta)} \zeta \mathrm{d} \lambda(\zeta)$ pour une mesure positive $\lambda$ portée par le complémentaire de $V$. Or $\mu$ étant ergodique, $\lambda$ est nécessairement la masse de Dirac en $\mu \in V$, ce qui donne la contradiction cherchée.

Une base dénombrable de tels voisinages $V_{n}$ de $\mu$ induit de cette manière des ensembles $\mu$-effilés $E_{n}$; or la convergence $\mathscr{G}^{\delta, y} \rightarrow \mu$ de l'énoncé a par définition lieu pour $y$ tendant coniquement vers n'importe quel point du complémentaire de $\bigcup_{n} \Lambda_{c}\left(E_{n}\right)$, lequel est $\mu_{o}$-négligeable par l'effilement des $E_{n}$.

\section{Principe du maximum}

L'objet de ce paragraphe est le principe du maximum, dans sa version pour les potentiels (cf [2], [10]). Là encore, une constante vient dilater l'inégalité finale, contrairement à la situation classique. Il est cependant à noter qu'une pareille notion de principe du maximum dilaté a déjà été envisagée (dans [7] que nous a indiqué A. Ancona, puis [16]).

THÉORÈme 6. - Supposons que $Y \subset X$ invariant par $\Gamma$ vérifie le principe des ombres pour $\Gamma$ en $\delta$. Soient $\mu$ et $\nu$ dans $\mathscr{C}(\Gamma, \delta)$, et $Z \subset Y$ invariant par $\Gamma$ tel que $\mu$ soit portée par $Z \cup \Lambda_{c}(Z)$. Si pour tout $z \in Z$ on a $\left\|\mu_{z}\right\| \leq\left\|\nu_{z}\right\|$, alors pour tout $y \in Y,\left\|\mu_{y}\right\| \leq C\left\|\nu_{y}\right\|$, où $C$ ne dépend que de $Y$ et de $\delta$. 
Démonstration. - Fixons $y \in Y$. Soit $Z^{*}$ donné par le lemme 1 avec $y$ à la place de $x$. Alors, en appliquant tour à tour le principe des ombres et l'hypothèse, $\left\|\mu_{y}\right\|=\mu_{y}\left(\bigcup_{z \in Z^{*}} \theta_{5 r}(y, z)\right) \leq e^{10 \delta r} \sum_{z \in Z^{*}}\left\|\mu_{z}\right\| e^{-\delta d(y, z)} \leq$ $e^{10 \delta r} \sum_{z \in Z^{*}}\left\|\nu_{z}\right\| e^{-\delta d(y, z)} \leq C \nu_{y}\left(\bigcup_{z \in Z^{*}} \theta_{r}(y, z)\right) \leq C\left\|\nu_{y}\right\|$.

Corollaire 2. - Supposons que $Y \subset X$ invariant par $\Gamma$ vérifie le principe des ombres pour $\Gamma$ en $\delta$. Soient $\mu$ et $\nu$ dans $\mathscr{C}(\Gamma, \delta)$, et $Z \subset Y$ invariant par $\Gamma$. Si pour tout $z \in Z$ on a $\left\|\mu_{z}\right\| \leq\left\|\nu_{z}\right\|$, alors pour tout $y \in Y,\left\|R_{Z} \mu_{y}\right\| \leq C\left\|\nu_{y}\right\|$, où $C$ ne dépend que de $Y$ et de $\delta$.

\section{La série de Poincaré comme noyau de Green}

Cette section est entièrement consacrée à la série de Poincaré $G^{\delta}(x, y)$, qui dans l'analogie que nous avons développée joue le rôle du noyau de Green.

On peut voir le résultat ci-dessous comme un lemme de l'ombre sur le quotient $Y / \Gamma$, et les inégalités (17) comme un raffinement des inégalités de Harnack (4) sous l'invariance par le groupe $\Gamma$.

THÉORÈme 7. - Supposons que $Y \subset X$ invariant par $\Gamma$ vérifie le principe des ombres pour $\Gamma$ en $\delta$. Il existe alors une constante $C \geq 1$ telle que pour tous $\mu \in \mathscr{C}(\Gamma, \delta)$ et $x, y \in Y$,

$$
\frac{1}{C} \frac{G^{\delta}(x, y)}{G^{\delta}(y, y)}\left\|\mu_{y}\right\| \leq \mu_{x}\left(\bigcup_{\gamma \in \Gamma} \vartheta_{r}(x, \gamma y)\right) \leq C \frac{G^{\delta}(x, y)}{G^{\delta}(y, y)}\left\|\mu_{y}\right\| .
$$

En particulier,

$$
\frac{1}{C} \frac{G^{\delta}(x, y)}{G^{\delta}(y, y)}\left\|\mu_{y}\right\| \leq\left\|\mu_{x}\right\| \leq C \frac{G^{\delta}(x, x)}{G^{\delta}(x, y)}\left\|\mu_{y}\right\| .
$$

Démonstration. - Le lemme 1 nous fournit $\Gamma^{*} y$ associé à $\Gamma y$ mis à la place du $Z$ de l'énoncé. On a alors (en notant $C_{0}=C(Y, \delta)$ et $\left.r=r(Y, \delta)\right)$ :

$$
\frac{1}{C_{0}} \sum_{\gamma \in \Gamma^{*}} e^{-\delta d(x, \gamma y)}\left\|\mu_{y}\right\| \leq \mu_{x}\left(\bigcup_{\gamma \in \Gamma} \vartheta_{r}(x, \gamma y)\right) \leq e^{10 \delta r} \sum_{\gamma \in \Gamma^{*}} e^{-\delta d(x, \gamma y)}\left\|\mu_{y}\right\| .
$$

En récrivant ces inégalités avec $\tilde{\mathscr{C}}^{\delta, y}$ au lieu de $\mu$, on obtient d'une part

$$
G^{\delta}(x, y)=\tilde{\mathscr{G}}_{x}^{\delta, y}\left(\bigcup_{\gamma \in \Gamma} \vartheta_{r}(x, \gamma y)\right) \leq e^{10 \delta r} \sum_{\gamma \in \Gamma^{*}} e^{-\delta d(x, \gamma y)} G^{\delta}(y, y),
$$

et d'autre part

$$
\frac{1}{C_{0}} \sum_{\gamma \in \Gamma^{*}} e^{-\delta d(x, \gamma y)} G^{\delta}(y, y) \leq G^{\delta}(x, y)
$$

TOME $139-2011-\mathrm{N}^{\mathrm{O}} 1$ 
En combinant ces inégalités, on obtient le résultat, avec $C=e^{10 \delta r} C_{0}$.

Le corollaire ci-dessous, qui dans la théorie du potentiel classique s'écrit avec $C=1$, permet de définir la «distance de Green » entre deux points $\Gamma x$ et $\Gamma y$ dans $Y / \Gamma$ par $-\log \frac{G^{\delta}(x, y)}{\sqrt{G^{\delta}(x, x) G^{\delta}(y, y)}}$ (avec l'ajustement que l'on voudra pour que cette expression définisse encore une distance stricto sensu lorsque $C>1$ ). La compactification de Martin apparaît alors comme la compactification de Busemann pour cette métrique (cf [3]).

Corollaire 3. - Supposons que $Y \subset X$ invariant par $\Gamma$ vérifie le principe des ombres pour $\Gamma$ en $\delta$. Il existe alors une constante $C \geq 1$ telle que pour tous $x, y, z \in Y$,

$$
G^{\delta}(x, y) G^{\delta}(y, z) \leq C G^{\delta}(y, y) G^{\delta}(x, z)
$$

Démonstration. - Cela découle de l'inégalité de gauche dans (17) avec $\mu=$ $\tilde{\mathscr{G}}^{\delta, z}$.

Nous obtenons l'estimée suivante pour la décroissance de la fonction de Green.

COROllaire 4. - Supposons que $Y \subset X$ invariant par $\Gamma$ vérifie le principe des ombres pour $\Gamma$ sur $\left[\delta(\Gamma), \delta_{0}\right]$. Il existe alors une constante $C>0$ telle que pour tous $\delta(\Gamma) \leq \sigma \leq \delta \leq \delta_{0}$, pour tous $x, y \in Y$,

$$
G^{\delta}(x, y) \leq C \sup _{z \in Y} G^{\sigma}(z, z) e^{-(\delta-\sigma) d(\Gamma x, \Gamma y)} .
$$

Démonstration. - On voit aisément que $G^{\delta}(x, y) \leq e^{-(\delta-\sigma) d(\Gamma x, \Gamma y)} G^{\sigma}(x, y)$. On applique ensuite le corollaire ci-dessus avec $\sigma$ au lieu de $\delta$ et $z=x$.

Le lemme suivant nous sera utile.

Lemme 5. - Supposons que $X_{\Gamma} \subset \bigcup_{y \in Y} B\left(y, r_{0}\right)$ où $Y \subset X_{\Gamma}$ et $r_{0}>0$. Pour tous $x, y \in X_{\Gamma}$, on $a[x y] \subset \bigcup_{z \in Y} B(z, r)$, où $r=r_{0}+2 \cosh ^{-1} \sqrt{2}$.

Démonstration. - Soient $\xi^{-}, \xi^{+}, \eta^{-}, \eta^{+} \in \Lambda(\Gamma)$ tels que $x \in\left(\xi^{-} \xi^{+}\right)$et $y \in$ $\left(\eta^{-} \eta^{+}\right)$. Par comparaison à la géométrie de $\mathbb{H}$, on voit que tout point de $[x y]$ est à distance au plus $\cosh ^{-1} \sqrt{2}$ de $\left[x \xi^{-}\right) \cup\left(\xi^{-} y\right]$ (cf [5]), et que tout point de $\left(\xi^{-} y\right]$ est à distance au plus $\cosh ^{-1} \sqrt{2}$ de $\left(\xi^{-} \eta^{-}\right) \cup\left(\eta^{-} y\right] \subset X_{\Gamma}$. Or $X_{\Gamma} \subset$ $\bigcup_{z \in Y} B\left(z, r_{0}\right)$, donc tout point de $[x y]$ est à distance inférieure strictement à $2 \cosh ^{-1} \sqrt{2}+r_{0}=r$ de $Y$.

Le théorème suivant fait songer à l'équation résolvante bien connue de la théorie classique (cf [10]), toujours à la constante $C$ près. 
THÉORÈme 8. - Supposons que $X_{\Gamma}$ vérifie le principe des ombres pour $\Gamma$ sur $\left[\delta(\Gamma), \delta_{0}\right]$ où $\delta_{0}>\delta(\Gamma)$. Soit $\lambda$ une mesure de Radon sur $X_{\Gamma} / \Gamma$ telle que pour une certaine constante $D>0$, pour tout $x \in X_{\Gamma}$,

$$
\frac{1}{D N(x)} \leq \lambda\left(B\left(\Gamma x, \frac{r_{0}}{2}\right)\right) \leq \lambda\left(B\left(\Gamma x, r_{0}\right)\right) \leq \frac{D}{N(x)}
$$

où $r_{0}=4 r\left(X_{\Gamma},\left[\delta(\Gamma), \delta_{0}\right]\right)$ et $N(x)=\sharp\left(\Gamma x \cap B\left(x, r_{0}\right)\right)$.

Il existe alors une constante $C>0$ telle que pour tous $\delta(\Gamma)<\sigma \leq \delta \leq \delta_{0}$, pour tous $x, y \in X_{\Gamma}$,

$$
\frac{1}{C}\left(G^{\sigma}(x, y)-G^{\delta}(x, y)\right) \leq(\delta-\sigma) \int_{X_{\Gamma} / \Gamma} G^{\sigma}(x, z) G^{\delta}(z, y) \mathrm{d} \lambda(z) \leq C\left(G^{\sigma}(x, y)-G^{\delta}(x, y)\right) .
$$

Démonstration. - Notons $r_{0}=4 r\left(X_{\Gamma},\left[\delta(\Gamma), \delta_{0}\right]\right), C_{0}=C\left(X_{\Gamma},\left[\delta(\Gamma), \delta_{0}\right]\right)$.

Commençons par discrétiser $X_{\Gamma}$ et $\lambda$ : prenons $Y \subset X_{\Gamma}$ invariant par $\Gamma$ tel que $Y / \Gamma$ soit un sous-ensemble $r_{0}$-séparé maximal de $X_{\Gamma} / \Gamma$; d'après les inégalités de Harnack (4) et compte tenu de (20), nous pouvons clairement nous ramener à $\lambda=\sum_{z \in Y / \Gamma} N(z)^{-1} \mathscr{D}_{z}$.

On peut également supposer que $\delta>\sigma$. Notons $\mu=\tilde{\mathscr{G}}^{\sigma, x}$, de sorte que l'on a $G^{\sigma}(x, y)=\left\|\mu_{y}\right\|$. Par le procédé d'intégration par parties, on vérifie aisément que $(\delta-\sigma) \int_{0}^{\infty} e^{-(\delta-\sigma) \rho} \mu_{y}(B(y, \rho)) \mathrm{d} \rho=G^{\delta}(x, y)$, d'où il vient :

$$
G^{\sigma}(x, y)-G^{\delta}(x, y)=(\delta-\sigma) \int_{0}^{\infty} e^{-(\delta-\sigma) \rho} \mu_{y}(X \backslash B(y, \rho)) \mathrm{d} \rho .
$$

Prenons $r=r_{0}+2 \cosh ^{-1} \sqrt{2}$, et notons $A_{n}=\{z \in Y \mid n r<d(y, z) \leq$ $(n+1) r\}$. On voit facilement que $X \backslash B(y, n r) \supset \bigcup_{z \in A_{n+1}} \theta_{r}(y, z)$ et que le recouvrement $\left\{\theta_{r}(y, z)\right\}_{z \in A_{n+1}}$ est à multiplicité bornée sur $\theta_{r}(y, z)$ par $\sharp(Y \cap B(z, 5 r))$. D'après le lemme 4 , ce nombre est majoré par $M N(z)$ où $M$ ne dépend que de $\Gamma$ et $\delta_{0}$.

De ces observations et du principe des ombres, il vient :

$$
\begin{aligned}
\int_{0}^{\infty} e^{-(\delta-\sigma) \rho} \mu_{y}(X \backslash B(y, \rho)) \mathrm{d} \rho & \geq r \sum_{n=1}^{\infty} e^{-(\delta-\sigma) n r} \mu_{y}(X \backslash B(y, n r)) \\
& \geq \frac{r}{M} \sum_{n=1}^{\infty} e^{-(\delta-\sigma) n r} \sum_{z \in A_{n+1}} N(z)^{-1} \mu_{y}\left(\theta_{r}(y, z)\right) \\
& \geq \frac{r}{M C_{0}} \sum_{n=1}^{\infty} \sum_{z \in A_{n+1}} N(z)^{-1}\left\|\mu_{z}\right\| e^{-\sigma d(y, z)} e^{-(\delta-\sigma) n r}
\end{aligned}
$$

TOME $139-2011-\mathrm{N}^{\mathrm{O}} 1$ 


$$
\begin{aligned}
& \geq \frac{r}{M C_{0}} \sum_{\substack{z \in Y \\
d(y, z)>2 r}} N(z)^{-1}\left\|\mu_{z}\right\| e^{-\delta d(y, z)} \\
& \geq \frac{r}{M C_{0}} \sum_{z \in Y / \Gamma} N(z)^{-1} G^{\sigma}(x, z) \sum_{\substack{\gamma \in \Gamma \\
d(z, \gamma y)>2 r}} e^{-\delta d(z, \gamma y)} .
\end{aligned}
$$

Or, étant donnés $z \in Y$ et $y \in X_{\Gamma}$, prenons $y^{\prime} \in Y$ tel que $4 r<d\left(z, y^{\prime}\right)<6 r$, ce qui est possible d'après le lemme 5. Alors $\gamma y \in \theta_{r}\left(z, y^{\prime}\right) \Rightarrow d(z, \gamma y)>2 r$. En utilisant une fois de plus le principe des ombres, on obtient

$$
\begin{aligned}
\sum_{d(z, \gamma y)>2 r} e^{-\delta d(z, \gamma y)} & \geq \sum_{\gamma \in \Gamma} e^{-\delta d(z, \gamma y)} \mathscr{D}_{\gamma y}\left(\Theta_{r}\left(z, y^{\prime}\right)\right) \geq \frac{1}{C_{0}} e^{-\delta d\left(z, y^{\prime}\right)} \sum_{\gamma \in \Gamma} e^{-\delta d\left(y^{\prime}, \gamma y\right)} \\
& \geq \frac{1}{C_{0}} e^{-2 \delta d\left(z, y^{\prime}\right)} \sum_{\gamma \in \Gamma} e^{-\delta d(z, \gamma y)} \geq \frac{1}{C_{0}} e^{-12 \delta_{0} r} G^{\delta}(z, y) .
\end{aligned}
$$

Avec ce qui précède et (22), on en déduit la seconde inégalité de (21).

Inversement, le lemme 5 permet de voir que $X_{\Gamma} \backslash B(y, n r) \subset \bigcup_{z \in A_{n-1}} \vartheta_{r}(y, z)$. Prenons $A_{n-1}^{\prime} \subset A_{n-1} r$-séparé maximal. Alors

$$
\begin{aligned}
\int_{0}^{\infty} e^{-(\delta-\sigma) \rho} \mu_{y}(X \backslash B(y, \rho)) \mathrm{d} \rho & \leq r \sum_{n=0}^{\infty} e^{-(\delta-\sigma) n r} \mu_{y}\left(X_{\Gamma} \backslash B(y, n r)\right) \\
& \leq r \sum_{n=0}^{\infty} e^{-(\delta-\sigma) n r} \sum_{z^{\prime} \in A_{n-1}^{\prime}} \mu_{y}\left(\theta_{2 r}\left(y, z^{\prime}\right)\right) \\
& \leq r e^{4 \delta_{0} r} \sum_{n=0}^{\infty} e^{-(\delta-\sigma) n r} \sum_{z^{\prime} \in A_{n-1}^{\prime}}\left\|\mu_{z^{\prime}}\right\| e^{-\sigma d\left(y, z^{\prime}\right)} \\
& \leq r e^{5 \delta_{0} r} \sum_{n=0}^{\infty} \sum_{z^{\prime} \in A_{n-1}^{\prime}}\left\|\mu_{z^{\prime}}\right\| e^{-\delta d\left(y, z^{\prime}\right)} \\
& \leq r e^{6 \delta_{0} r} \sum_{n=0}^{\infty} \sum_{z \in \Gamma z^{\prime} \cap B\left(z^{\prime}, r_{0}\right)} N(z)^{-1}\left\|\mu_{z}\right\| e^{-\delta d(y, z)} \\
& \leq r e^{6 \delta_{0} r} \sum_{n=0}^{\infty} \sum_{z \in A_{n-2} \cup A_{n-1} \cup A_{n}} N(z)^{-1}\left\|\mu_{z}\right\| e^{-\delta d(y, z)} \\
& \leq 3 r e^{6 \delta_{0} r} \sum_{z \in Y} N(z)^{-1}\left\|\mu_{z}\right\| e^{-\delta d(y, z)} .
\end{aligned}
$$

Après regroupement selon $\Gamma$, cela achève, avec (22), la preuve de la première inégalité de (21). 
Le corollaire suivant vient compléter le corollaire 4 .

Corollaire 5. - Supposons que $X_{\Gamma}$ vérifie le principe des ombres pour $\Gamma$ sur $\left[\delta(\Gamma), \delta_{0}\right]$. Il existe alors une constante $C>0$ telle que $\left.\left.\forall \delta \in\right] \delta(\Gamma), \delta_{0}\right], \forall x \in X_{\Gamma}$,

$$
\max \left\{1, e^{-\delta r_{0}} N(x)\right\} \leq G^{\delta}(x, x) \leq \frac{C}{\delta-\delta(\Gamma)} N(x),
$$

ò̀ $N(x)=\sharp\left(\Gamma x \cap B\left(x, r_{0}\right)\right)$ et $r_{0}=4 r\left(X_{\Gamma},\left[\delta(\Gamma), \delta_{0}\right]\right)$.

Démonstration. - Appliquons le théorème en prenant $x=y$. On a alors

$(\delta-\sigma) \frac{e^{-2 \delta r_{0}}}{D N(x)} G^{\sigma}(x, x) G^{\delta}(x, x) \leq(\delta-\sigma) \int_{X_{\Gamma} / \Gamma} G^{\sigma}(x, z) G^{\delta}(z, x) \mathrm{d} \lambda(z) \leq$ $C G^{\sigma}(x, x)$. Après simplification, on peut faire tendre $\sigma$ vers $\delta(\Gamma)$ pour obtenir la seconde inégalité ci-dessus, la première étant quant à elle triviale.

\section{Mesure harmonique}

Supposons que $X_{\Gamma}$ vérifie le principe des ombres en $\delta$ et que $G^{\delta}<\infty$. Le théorème 3 (voir sa démonstration) définit l'opération de réduite $R_{Z}$ des densités de $\mathscr{C}(\Gamma, \delta)$ sur n'importe quel sous-ensemble $Z$ de $X_{\Gamma}$ invariant par $\Gamma$; nous nous référons ici à cette construction, moyennant une précision qui sera donnée dans la démonstration ci-dessous.

Nous définissons à présent la mesure (pseudo - ) harmonique $H_{x}^{\delta, R}$ du point $\bar{x}=\Gamma x \in X_{\Gamma} / \Gamma$ dans la boule $B(\bar{x}, R)$ par

$$
R_{X_{\Gamma} \backslash B(\bar{x}, R)} \tilde{\mathscr{G}}^{\delta, x}=\int_{X_{\Gamma} / \Gamma} \tilde{\mathscr{G}}^{\delta, y} \mathrm{~d} H_{x}^{\delta, R}(y) .
$$

Proposition 2. - Supposons que $X_{\Gamma}$ vérifie le principe des ombres pour $\Gamma$ $\operatorname{sur}\left[\delta(\Gamma), \delta_{0}\right]$ où $\delta_{0}>\delta(\Gamma)$.

i) Il existe une constante $C>0$ ne dépendant que de $\delta_{0}$ telle que pour $\mu \in \mathscr{C}(\Gamma, \delta), x \in X_{\Gamma}$ et $R>0$,

$$
\begin{gathered}
\int_{X_{\Gamma} / \Gamma}\left\|\mu_{y}\right\| \mathrm{d} H_{x}^{\delta, R}(y) \leq C\left\|\mu_{x}\right\| \\
\text { et, si } \mu_{o}(B(\Gamma x, R))=0, \quad\left\|\mu_{x}\right\| \leq C \int_{X_{\Gamma} / \Gamma}\left\|\mu_{y}\right\| \mathrm{d} H_{x}^{\delta, R}(y) .
\end{gathered}
$$

ii) Il existe $R_{0}>0$ ne dépendant que de $\delta_{0}$ tel que pour tous $x \in X_{\Gamma}$ et $R>0$, le support de $H_{x}^{\delta, R}$ est contenu dans la couronne de centre $\bar{x}=\Gamma x$ définie par $\left\{\bar{y} \in X_{\Gamma} / \Gamma \mid R \leq d(\bar{x}, \bar{y})<R+R_{0}\right\}$.

iii) Si $\sigma \leq \delta$, alors $H_{x}^{\delta, R} \leq e^{-(\delta-\sigma) R} H_{x}^{\sigma, R}$. 
Démonstration. - D'après (24) et le théorème 3 , pour tout $z \in X_{\Gamma}$,

$$
\int_{X_{\Gamma} / \Gamma} G^{\delta}(y, z) \mathrm{d} H_{x}^{\delta, R}(y)=\left\|R_{X_{\Gamma} \backslash B(\bar{x}, R)} \tilde{\mathscr{G}}^{\delta, x}\right\| \leq C G^{\delta}(x, z)
$$

(on rappelle que $G^{\delta}$ est symétrique), et pour tout $z \notin B(\bar{x}, R)$,

$$
G^{\delta}(x, z) \leq C \int_{X_{\Gamma} / \Gamma} G^{\delta}(y, z) \mathrm{d} H_{x}^{\delta, R}(y) .
$$

On en déduit le i) du lemme pour $\mu$ portée par $X_{\Gamma}$; le cas général suit grâce au théorème 4 .

Pour obtenir ii), revenons à la définition de la réduite dans la démonstration du théorème 3 ; et appelons ici $R^{\prime}$ le rayon $R$ alors introduit, afin d'éviter une confusion. Nous choisissons $Y^{\prime}$ comme alors, mais nous imposerons $R^{\prime}>$ $r+2 \cosh ^{-1} \sqrt{2}$, et pour $Z^{\prime}$ nous prendrons plutôt $Z^{\prime}=Y^{\prime} \backslash \Gamma B(x, R)$, ce qui nous donne dans (ii) que le support de $H_{x}^{\delta, R}$ est hors de $B(\bar{x}, R)$, sans affecter le reste. Il est d'autre part facile de voir que si $\Gamma x \cap \pi\left(\phi_{y}\right) \neq \varnothing$, alors $d(\Gamma x, y)<R+R_{0}$ par définition de $\phi_{y}$, avec $R_{0}=R^{\prime}+2 r+2 \cosh ^{-1} \sqrt{2}$ : en effet, $\Gamma x \cap \pi\left(\phi_{y^{\prime}}\right) \neq \varnothing$ pour un $y^{\prime} \in B(y, r)$; autrement dit, il existe un rayon $(\eta \gamma x]$ ne rencontrant plus d'autre boule de rayon $R^{\prime}$ centrée en $Z^{\prime}$ après $B\left(y^{\prime}, R^{\prime}\right)$; le point du bord où le rayon sort de cette boule appartient à une boule $B\left(x^{\prime}, r+2 \cosh ^{-1} \sqrt{2}\right)$ avec $x^{\prime} \in Y^{\prime}$ d'après le lemme 5 , mais $x^{\prime} \notin Z^{\prime}$ et donc $d\left(\Gamma x, x^{\prime}\right)<R$; en fin de compte, on trouve le résultat annoncé.

iii) est immédiat.

\section{Identification géométrique de la frontière de Martin dans le cas hyperbolique}

Munis de tout le matériel exposé dans les sections précédentes, nous sommes maintenant en mesure de reprendre dans notre cadre les arguments de [1] (cf aussi [2]), afin de montrer, sous l'hypothèse que $X_{\Gamma}$ vérifie le principe des ombres et que $X_{\Gamma} / \Gamma$ est hyperbolique au sens de Gromov (voir [12],[11], [2]), que la frontière de Martin $\mathcal{F}(\Gamma, \delta)$ pour $\delta>\delta(\Gamma)$ est réduite à sa partie minimale et s'identifie au bord à l'infini de $X_{\Gamma} / \Gamma$.

Les exemples d'une telle situation abondent : il suffit (grâce au théorème 1) de prendre pour $\Gamma$ un sous-groupe distingué d'un groupe convexe-cocompact $\Gamma_{0}$ dont le quotient $\Gamma_{0} / \Gamma$ est un groupe hyperbolique, ce qui est assurément loisible lorsque $\Gamma_{0}$ est un groupe libre comme il est courant d'en obtenir par les constructions ordinaires.

La démonstration suivra celle de [1], et tout notre apport consiste à montrer comment il est possible ici d'en conserver les arguments généraux.

La clef de cette approche est la propriété suivante de multiplicativité de la fonction de Green le long d'une géodésique. Rappelons que l'inégalité de gauche 
ci-dessous est générale (voyez (18)). L'hypothèse essentielle $\delta>\delta(\Gamma)$ correspond à l'hypothèse de coercivité faible au sens d'Ancona. Une légère différence entre notre formulation et celle de [2] provient de ce que nous n'avons pas fait ici d'hypothèse de géométrie bornée sur $X_{\Gamma} / \Gamma$ et que par conséquent $G^{\delta}(x, x)$ n'est pas uniformément bornée en général.

Proposition 3. - Supposons que $X_{\Gamma}$ vérifie le principe des ombres sur $\left[\delta(\Gamma), \delta_{0}\right]$ où $\delta_{0}>\delta(\Gamma)$, et que $X_{\Gamma} / \Gamma$ est hyperbolique au sens de Gromov.

Soit $\left.\delta \in] \delta(\Gamma), \delta_{0}\right]$. Il existe $C>0$ telle que si $x, y, z$ sont alignés dans cet ordre sur un segment géodésique minimisant dans $X_{\Gamma} / \Gamma$, alors

$$
\frac{1}{C} G^{\delta}(x, y) G^{\delta}(y, z) \leq G^{\delta}(x, z) G^{\delta}(y, y) \leq C G^{\delta}(x, y) G^{\delta}(y, z)
$$

Démonstration. - Les constantes $C_{0}, C_{1}, \ldots$ ci-dessous ne dépendront que de $X_{\Gamma} / \Gamma$ et $\operatorname{de} \delta$.

On considère une $\Phi$-chaîne au sens de [2], c'est-à-dire des points $x_{0}=$ $x, x_{1}, \ldots, x_{n}=z$ dans $X_{\Gamma} / \Gamma$ répartis dans cet ordre sur le segment $\left[x_{0} x_{n}\right]$ tels que $d\left(x_{i}, x_{i+1}\right)=\rho$ où $\rho>0$ vaut quatre fois la (une) constante d'hyperbolicité de $X_{\Gamma} / \Gamma$ (habituellement notée $\delta$ ), et une suite décroissante d'ouverts $X_{\Gamma} \supset U_{0} \supset U_{1} \supset \cdots \supset U_{n}$ donnés par $U_{k}=\left\{z \in X_{\Gamma} / \Gamma \mid\left\langle z, x_{k}^{\prime}\right\rangle_{x_{0}}>k \rho\right\}$, où $x_{k}^{\prime}$ est le milieu de $\left[x_{k} x_{k+1}\right]$ et $\langle.,$.$\rangle . désigne le produit de Gromov : \langle a, b\rangle_{c}=$ $\frac{1}{2}[d(a, c)+d(c, b)-d(a, b)]$.

Prenons $\sigma=\frac{\delta(\Gamma)+2 \delta}{3}$. La méthode consiste à montrer d'abord :

$$
\forall y \in U_{k}, \quad G^{\delta}\left(x_{0}, y\right) G^{\delta}\left(x_{k}, x_{k}\right) \leq C_{0} G^{\delta}\left(x_{0}, x_{k}\right) G^{\sigma}\left(x_{k}, y\right)
$$

Fixons $R>0$ assez grand pour que $e^{-(\delta-\sigma) R+4 \delta \rho} K^{2} \leq 1$ où $K$ est la constante $C$ de la proposition 2.

Par l'hyperbolicité de $X_{\Gamma} / \Gamma$, il existe $D>R$ ne dépendant que de $X_{\Gamma} / \Gamma$ et de $R$ telle que si $y \in U_{k+1}$ et $d\left(y, x_{k+1}\right)>D$, alors $B\left(y, R+R_{0}\right) \subset U_{k}$, où $R_{0}$ est le rayon donné par la proposition 2 (ii).

Nous allons montrer (28) pour $C_{0}=e^{2 \delta D}$, par récurrence sur $k$. Le cas $k=0$ est trivial. Supposant (28) vraie pour $k$, considérons $y \in U_{k+1}$. Si $d\left(y, x_{k+1}\right)>$ 
$D$, alors $\operatorname{supp}\left(H_{y}^{\delta, R}\right) \subset B\left(y, R+R_{0}\right) \subset U_{k}$ grâce à la proposition 2 (ii), puis (proposition 2 (i) et (4))

$G^{\delta}\left(x_{0}, y\right) G^{\delta}\left(x_{k+1}, x_{k+1}\right) \leq e^{2 \delta \rho} K \int_{U_{k}} G^{\delta}\left(x_{0}, y^{\prime}\right) \mathrm{d} H_{y}^{\delta, R}\left(y^{\prime}\right) G^{\delta}\left(x_{k}, x_{k}\right)$

( par (28)) $\leq e^{2 \delta \rho} K C_{0} G^{\delta}\left(x_{0}, x_{k}\right) \int G^{\sigma}\left(x_{k}, y^{\prime}\right) \mathrm{d} H_{y}^{\delta, R}\left(y^{\prime}\right)$

(proposition 2 (iii)) $\leq e^{-(\delta-\sigma) R+2 \delta \rho} K C_{0} G^{\delta}\left(x_{0}, x_{k}\right) \int G^{\sigma}\left(x_{k}, y^{\prime}\right) \mathrm{d} H_{y}^{\sigma, R}\left(y^{\prime}\right)$

(proposition 2 (i)) $\leq e^{-(\delta-\sigma) R+2 \delta \rho} K^{2} C_{0} G^{\delta}\left(x_{0}, x_{k}\right) G^{\sigma}\left(x_{k}, y\right)$

((4) et choix de $R$ ) $\leq C_{0} G^{\delta}\left(x_{0}, x_{k+1}\right) G^{\sigma}\left(x_{k+1}, y\right)$.

Si au contraire $d\left(y, x_{k+1}\right) \leq D$, on a, en utilisant (4),

$$
G^{\delta}\left(x_{0}, y\right) G^{\delta}\left(x_{k+1}, x_{k+1}\right) \leq e^{2 \delta D} G^{\delta}\left(x_{0}, x_{k+1}\right) G^{\sigma}\left(x_{k+1}, y\right) .
$$

Nous avons donc établi (28) à l'ordre $k+1$ à partir de $k$, et finalement pour tout $k$.

Nous allons maintenant déduire (27) de (28).

Par le théorème 3 , il existe une mesure positive finie $b$ sur $X_{\Gamma} / \Gamma$ telle que

$$
\left\|R_{X_{\Gamma} \backslash \Gamma U_{k}} \tilde{\mathscr{G}}_{y}^{\delta, x_{n}}\right\|=\int G^{\delta}(y, z) \mathrm{d} b(z)
$$

(dans notre analogie, $b$ est (proportionnelle à) la balayée de la masse de Dirac en $x_{n}$ sur le complémentaire de $U_{k}$ ). De la même façon que dans la démonstration de la proposition 2 (ii), on voit facilement que $b$ est portée par un $R_{0}$-voisinage uniforme du bord de $U_{k}$, c'est-à-dire $\partial_{R_{0}} U_{k}=\left\{z \in U_{k} \mid d\left(z, \partial U_{k}\right)<R_{0}\right\}$.

En utilisant le théorème 3 (vu que $x_{0} \in X_{\Gamma} \backslash \Gamma U_{k}$ ) puis (28), il vient

$$
\begin{aligned}
G^{\delta}\left(x_{0}, x_{n}\right) G^{\delta}\left(x_{k}, x_{k}\right) & \leq C_{1} \int_{\partial_{R_{0}} U_{k}} G^{\delta}\left(x_{0}, z\right) \mathrm{d} b(z) G^{\delta}\left(x_{k}, x_{k}\right) \\
& \leq C_{2} G^{\delta}\left(x_{0}, x_{k}\right) \int_{\partial_{R_{0}} U_{k}} G^{\sigma}\left(x_{k}, z\right) \mathrm{d} b(z) .
\end{aligned}
$$

Or avec l' « équation résolvante » (21), on voit facilement que pour tout $z \in$ $X_{\Gamma} / \Gamma$,

$$
G^{\sigma}\left(x_{k}, z\right) \leq C_{3} \int_{X_{\Gamma} / \Gamma} G^{\sigma}\left(x_{k}, y\right) G^{\delta}(y, z) \mathrm{d} \lambda(y)
$$

(notez que $G^{\delta}\left(x_{k}, z\right) \leq C_{3}^{\prime} \int_{B\left(x_{k}, r_{0}\right)} G^{\sigma}\left(x_{k}, y\right) G^{\delta}(y, z) \mathrm{d} \lambda(y)$ grâce à (4), (20) et (23)). Par suite,

$$
G^{\delta}\left(x_{0}, x_{n}\right) G^{\delta}\left(x_{k}, x_{k}\right) \leq C_{4} G^{\delta}\left(x_{0}, x_{k}\right) \int_{X_{\Gamma} / \Gamma} \mathrm{d} \lambda(y) G^{\sigma}\left(x_{k}, y\right) \int_{\partial_{R_{0}} U_{k}} G^{\delta}(y, z) \mathrm{d} b(z) .
$$


Si on applique de nouveau (28), mais pour la chaîne inverse $x_{n}, \ldots, x_{0}$, avec $\left(X_{\Gamma} / \Gamma\right) \backslash U_{n}, \ldots,\left(X_{\Gamma} / \Gamma\right) \backslash U_{0}$ au lieu de $U_{0}, \ldots, U_{n}$, on obtient pour tout $y \notin U_{k}$,

$$
G^{\delta}\left(x_{n}, y\right) G^{\delta}\left(x_{k}, x_{k}\right) \leq C_{5} G^{\delta}\left(x_{k}, x_{n}\right) G^{\sigma}\left(x_{k}, y\right) .
$$

Avec les inégalités de Harnack (4), on en déduit que pour tout $y \in \partial_{R_{0}} U_{k}$,

$$
G^{\delta}\left(x_{n}, y\right) G^{\delta}\left(x_{k}, x_{k}\right) \leq C_{5} e^{(\delta+\sigma) R_{0}} G^{\delta}\left(x_{k}, x_{n}\right) G^{\sigma}\left(x_{k}, y\right) .
$$

En vertu de (30) et du théorème 3 , il en résulte que pour tout $y \in \partial_{R_{0}} U_{k}$,

$$
\int_{\partial_{R_{0}} U_{k}} G^{\delta}(y, z) \mathrm{d} b(z) G^{\delta}\left(x_{k}, x_{k}\right) \leq C_{6} G^{\delta}\left(x_{k}, x_{n}\right) G^{\sigma}\left(x_{k}, y\right) .
$$

Avec (31), il vient enfin que pour tout $y \in \partial_{R_{0}} U_{k}$,

$\int_{\partial_{R_{0}} U_{k}} G^{\delta}(y, z) \mathrm{d} b(z) G^{\delta}\left(x_{k}, x_{k}\right) \leq C_{7} G^{\delta}\left(x_{k}, x_{n}\right) \int_{X_{\Gamma} / \Gamma} G^{\sigma}\left(x_{k}, y^{\prime}\right) G^{\delta}\left(y, y^{\prime}\right) \mathrm{d} \lambda\left(y^{\prime}\right)$.

En appliquant alors le théorème 6 (principe du maximum), puis encore (21), il s'ensuit que pour tout $y \in X_{\Gamma} / \Gamma$,

$$
\begin{aligned}
\int_{\partial_{R_{0}} U_{k}} G^{\delta}(y, z) \mathrm{d} b(z) G^{\delta}\left(x_{k}, x_{k}\right) & \leq C_{8} G^{\delta}\left(x_{k}, x_{n}\right) \int_{X_{\Gamma} / \Gamma} G^{\sigma}\left(x_{k}, y^{\prime}\right) G^{\delta}\left(y, y^{\prime}\right) \mathrm{d} \lambda\left(y^{\prime}\right) \\
& \leq C_{9} G^{\delta}\left(x_{k}, x_{n}\right) G^{\sigma}\left(x_{k}, y\right) .
\end{aligned}
$$

Avec (32), cela entraîne

$$
G^{\delta}\left(x_{0}, x_{n}\right) G^{\delta}\left(x_{k}, x_{k}\right)^{2} \leq C_{10} G^{\delta}\left(x_{0}, x_{k}\right) G^{\delta}\left(x_{k}, x_{n}\right) \int_{X_{\Gamma} / \Gamma} G^{\sigma}\left(x_{k}, y\right)^{2} \mathrm{~d} \lambda(y) .
$$

Soit $\sigma^{\prime}=\frac{2 \delta(\Gamma)+\delta}{3}$. D'après $(21)$, on a

$$
\int_{X_{\Gamma} / \Gamma} G^{\sigma}\left(x_{k}, y\right)^{2} \mathrm{~d} \lambda(y) \leq \int_{X_{\Gamma} / \Gamma} G^{\sigma^{\prime}}\left(x_{k}, y\right) G^{\sigma}\left(x_{k}, y\right) \mathrm{d} \lambda(y) \leq C_{11} G^{\sigma^{\prime}}\left(x_{k}, x_{k}\right) .
$$

Pour finir, (23) montre que $G^{\sigma^{\prime}}\left(x_{k}, x_{k}\right) \leq C_{12} G^{\delta}\left(x_{k}, x_{k}\right)$. Avec (34) et (33), la démonstration est bien terminée.

En poursuivant le programme de [1] (cf aussi [2]), nous établissons à partir de la proposition précédente l'identification géométrique de la frontière de Martin avec le bord à l'infini de $X_{\Gamma} / \Gamma$ supposé hyperbolique. A ce stade, la démonstration ne diffère plus guère des arguments généraux donnés dans [2], et sera simplement esquissée pour la commodité du lecteur. 
THÉORÈme 9. - Supposons que $X_{\Gamma}$ vérifie le principe des ombres sur $\left[\delta(\Gamma), \delta_{0}\right]$ où $\delta_{0}>\delta(\Gamma)$, et que $X_{\Gamma} / \Gamma$ est hyperbolique au sens de Gromov.

Soit $\left.\delta \in] \delta(\Gamma), \delta_{0}\right]$. Alors la frontière de Martin $\mathcal{F}(\Gamma, \delta)$ pour les densités conformes invariantes par $\Gamma$ de dimension $\delta$ est réduite à la frontière minimale (c'est-à-dire à l'ensemble $\mathcal{E}(\Gamma, \delta)$ des densités ergodiques) et s'identifie à la frontière idéale de $X_{\Gamma} / \Gamma$ par l'application bijective $\partial\left(X_{\Gamma} / \Gamma\right) \rightarrow \mathcal{E}(\Gamma, \delta), \zeta \mapsto$ $\mathscr{G}^{\delta, \zeta}=\lim _{X_{\Gamma} / \Gamma \ni y \rightarrow \zeta} \mathscr{G}^{\delta, y}$.

Notons encore cette estimation remarquable de $\left\|\mathscr{G}_{x}^{\delta, \zeta}\right\|$ pour $x$ sur un rayon géodésique minimisant $[o \zeta)$ dans $X_{\Gamma} / \Gamma$ :

$$
\frac{G^{\delta}(x, x)}{C G^{\delta}(o, x)} \leq\left\|\mathscr{G}_{x}^{\delta, \zeta}\right\| \leq \frac{C G^{\delta}(x, x)}{G^{\delta}(o, x)} \quad \text { où } C \text { ne dépend que de } X_{\Gamma} / \Gamma \text { et } \delta \text {. }
$$

(On applique (27) avec $o$ à la place de $x, x$ à la place de $y$, et $z$ tendant vers $\zeta$ après avoir tout divisé par $G^{\delta}(o, x) G^{\delta}(o, z)$.)

Démonstration. - On considère, pour $\zeta \in \partial\left(X_{\Gamma} / \Gamma\right)$, l'ensemble $\mathcal{H}_{\zeta}$ des $\mu \in$ $\mathscr{C}(\Gamma, \delta)$ pour lesquelles tout point $\zeta^{\prime} \in \partial\left(X_{\Gamma} / \Gamma\right) \backslash\{\zeta\}$ admette un voisinage $V$ dans $\overline{X_{\Gamma} / \Gamma}$ tel que $\left\|\mu_{x}\right\| \leq C G^{\delta}(o, x)$ pour tout $x \in X_{\Gamma} / \Gamma \cap V$ et une certaine constante $C$ ne dépendant pas de $x$.

Prenons $\zeta \in \partial\left(X_{\Gamma} / \Gamma\right)$, fixons un rayon géodésique minimisant $g$ du point $\bar{o}=\Gamma o$ à $\zeta$, et reprenons les notations de la démonstration précédente avec $x_{0}=\bar{o}, x_{1}, \ldots$ équirépartis sur $g$ avec $d\left(x_{i}, x_{i+1}\right)=\rho$, et les ouverts $U_{k}$, dont les adhérences forment une base de voisinages de $\zeta$. La proposition précédente nous donne la double inégalité (27) avec $y=x_{k}, x \notin U_{k}$ et $z \in U_{k+1}$, vu qu'un (tout) segment géodésique de $x$ à $z$ passe à distance uniformément bornée de $y$ selon l'hypothèse d'hyperbolicité. En la divisant par celle obtenue en faisant $x=\bar{o}$, il vient, pour tous $k \in \mathbb{N}, x \notin U_{k}$ et $z \in U_{k+1}$,

$$
\frac{1}{C^{2}}\left\|\mathscr{G}_{x}^{\delta, x_{k}}\right\| \leq\left\|\mathscr{G}_{x}^{\delta, z}\right\| \leq C^{2}\left\|\mathscr{G}_{x}^{\delta, x_{k}}\right\|
$$

Si $\mu \in \mathcal{F}(\Gamma, \delta)$ est limite d'une suite de points de $X_{\Gamma} / \Gamma$ qui converge également vers le point $\zeta$ sur la frontière idéale, il suit de (35), que pour tous $k \in \mathbb{N}$, $x \notin U_{k}$,

$$
\frac{1}{C^{2}}\left\|\mathscr{G}_{x}^{\delta, x_{k}}\right\| \leq\left\|\mu_{x}\right\| \leq C^{2}\left\|\mathscr{G}_{x}^{\delta, x_{k}}\right\|
$$

d'où $\mu \in \mathcal{H}_{\zeta}$.

Inversement, si $\mu \in \mathscr{H}_{\zeta}$, alors la réduite de $\mu$ sur le complémentaire de $U_{k}$ est par définition de $\mathcal{H}_{\zeta}$ un potentiel (c'est-à-dire une densité à support dans $X_{\Gamma}$, ce qu'on voit en appliquant le théorème 2$)$; par intégration de (35), il en résulte que $\mu$ satisfait à (36). 
Il est alors facile de voir que $\mathcal{H}_{\zeta}$ contient exactement un point (l'unicité repose sur le corollaire 1 ), puis que l'identification des frontières idéale et de Martin a lieu. Le lecteur pourra trouver le détail de ces arguments dans [2] au chapitre $\mathrm{V}$, théorème 6.2 .

\section{Un problème ouvert}

L'étude précédente a montré que la série de Poincaré ressemble fort à un noyau de Green, et même, en faisant varier la dimension $\delta$ à une résolvante (localement bien sûr). Aussi la question se pose-t-elle de savoir s'il existe un véritable noyau au sens de la théorie du potentiel (voir [10]) $\mathbf{G}^{\delta}(x, y)$ par rapport à une certaine mesure sur $X_{\Gamma} / \Gamma$ tel que $\mathbf{G}^{\delta}(x, y) \sim G^{\delta}(x, y)$ lorsque $d_{\Gamma}(x, y) \rightarrow \infty$ (de sorte que les harmoniques seraient conservées, ainsi que la frontière de Martin). Par exemple, dans le cadre classique où $X$ est le demi-plan de Poincaré et $\Lambda(\Gamma)$ le cercle à l'infini, on peut proposer $\mathbf{G}^{1}(x, y)=\sum_{\gamma \in \Gamma}-\log \tanh \left(\frac{d(x, \gamma y)}{2}\right)$ (c'est le noyau lié au laplacien).

Remarquons que nos énoncés paraissent issus d'une discrétisation grossière d'un bon noyau $\mathbf{G}^{\delta}(x, y)$, et qu'en effet nous avons souvent recouru à une discrétisation dans nos démonstrations. Pourtant, on sait qu'il est possible, dans le cadre classique, de discrétiser le laplacien sans inconvénient (voir [4] qui fait suite à [2] et [14]).

Il est à noter enfin que ce problème n'est pas résolu par la méthode de [9], bien que le thème commun soit de rapprocher mesures conformes et mesures harmoniques.

\section{BIBLIOGRAPHIE}

[1] A. AnCona - « Negatively curved manifolds, elliptic operators, and the Martin boundary », Ann. of Math. 125 (1987), p. 495-536.

[2] _ _ « Théorie du potentiel sur les graphes et les variétés », in École d'été de Probabilités de Saint-Flour XVIII-1988, Lecture Notes in Math., vol. 1427, Springer, 1990, p. 1-112.

[3] W. BALlmanN - Lectures on spaces of nonpositive curvature, DMV Seminar, vol. 25, Birkhäuser, 1995.

[4] W. Ballmann \& F. LEDRAPPIER - «Discretization of positive harmonic functions on Riemannian manifolds and Martin boundary », in Actes de la Table Ronde de Géométrie Différentielle (Luminy, 1992), Sémin. Congr., vol. 1, Soc. Math. France, 1996, p. 77-92.

[5] A. F. BEARDON - The geometry of discrete groups, Graduate Texts in Math., vol. 91, Springer, 1983.

TOME $139-2011-\mathrm{N}^{\mathrm{O}} 1$ 
[6] M. Bourdon - «Structure conforme au bord et flot géodésique d'un CAT(-1)-espace », Enseign. Math. 41 (1995), p. 63-102.

[7] G. Choquet - «Les noyaux réguliers en théorie du potentiel », C. $R$. Acad. Sci. Paris 243 (1956), p. 635-638.

[8] _ Lectures on analysis. Vol. II : Representation theory, Edited by J. Marsden, T. Lance and S. Gelbart, W. A. Benjamin, Inc., New YorkAmsterdam, 1969.

[9] C. Connell \& R. MuchniK - «Harmonicity of quasiconformal measures and Poisson boundaries of hyperbolic spaces », Geom. Funct. Anal. 17 (2007), p. 707-769.

[10] C. Dellacherie \& P.-A. Meyer - Probabilities and potential, NorthHolland Mathematics Studies, vol. 29, North-Holland Publishing Co., 1978.

[11] E. Ghys \& P. DE LA HARPe (éds.) - Sur les groupes hyperboliques d'après Mikhael Gromov, Progress in Math., vol. 83, Birkhäuser, 1990.

[12] M. Gromov - «Hyperbolic groups », in Essays in group theory, Math. Sci. Res. Inst. Publ., vol. 8, Springer, 1987, p. 75-263.

[13] F. LEDRAPPIER - « Harmonic measures and Bowen-Margulis measures », Israel J. Math. 71 (1990), p. 275-287.

[14] T. Lyons \& D. Sullivan - « Function theory, random paths and covering spaces », J. Differential Geom. 19 (1984), p. 299-323.

[15] R. S. Martin - « Minimal positive harmonic functions », Trans. Amer. Math. Soc. 49 (1941), p. 137-172.

[16] M. Oнtsuka - «Les relations entre certains principes en théorie du potentiel », Proc. Japan Acad. 33 (1957), p. 37-40.

[17] S. J. Patterson - « The limit set of a Fuchsian group», Acta Math. 136 (1976), p. 241-273.

[18] T. Roblin - « Sur la fonction orbitale des groupes discrets en courbure négative », Ann. Inst. Fourier (Grenoble) 52 (2002), p. 145-151.

[19] — , «Ergodicité et unique ergodicité du feuilletage horosphérique, mélange du flot géodésique et équidistributions diverses dans les groupes discrets en courbure négative», Mém. Soc. Math. Fr. 95 (2003).

[20] _ _ « Un théorème de Fatou pour les densités conformes avec applications aux revêtements galoisiens en courbure négative », Israel J. Math. 147 (2005), p. 333-357.

[21] D. Sullivan - « The density at infinity of a discrete group of hyperbolic motions », Publ. Math. I.H.É.S. 50 (1979), p. 171-202.

[22] _ _ «elated aspects of positivity in Riemannian geometry », J. Differential Geom. 25 (1987), p. 327-351. 